\title{
High time resolution observations of solar $\mathrm{H} \alpha$ flares. I. ${ }^{\star}$
}

\author{
K. Radziszewski ${ }^{1}$, P. Rudawy ${ }^{1}$, and K. J. H. Phillips ${ }^{2}$ \\ 1 Astronomical Institute of Wrocław University, 51-622 Wrocław, ul. Kopernika 11, Poland \\ e-mail: [radziszewski; rudawy] @astro.uni.wroc.pl \\ 2 Mullard Space Science Laboratory, Holmbury St Mary, Dorking, Surrey RH5 6NT, UK \\ e-mail: kennethjhphillips@yahoo.com
}

Received 18 May 2005 / Accepted 5 September 2006

\section{ABSTRACT}

\begin{abstract}
We present here the first results of a search for fast changes of solar flare $\mathrm{H} \alpha$ emission correlated in time with variations of hard X-ray fluxes recorded with the Reuven Ramaty High Energy Solar Spectroscopic Imager (RHESSI). Using the Large Coronagraph $(L C)$, Multi-Channel Subtractive Double Pass (MSDP) spectrograph and the Solar Eclipse Coronal Imaging System (SECIS) at the Białkow Observatory, we collected a total of 23 sets of high time resolution (0.04-0.075 s) observations of H $\alpha$ bright flare kernels during two observational seasons (2003 and 2004). For the first time, several-minute-long high-resolution observations of the $\mathrm{H}$-alpha line profiles as well as simultaneous two-dimensional images have been obtained with time resolution much better than $1 \mathrm{~s}$. Detailed observations of four flares recorded on 2003 July 16 and 2004 April 23 are described. We found examples of good time correlations between X-ray flux variations and variations of the $\mathrm{H} \alpha$ emission of the selected bright flaring kernels. In some events particularly, small but impulsive variations of the hard X-ray (20-50 keV) flux and of the $\mathrm{H} \alpha$ emission are well correlated. The $\mathrm{H} \alpha$ emission follows the 20-50 keV X-ray emission by $\sim 2 \mathrm{~s}$ and $\sim 3 \mathrm{~s}$ for two of the flares, longer for a third. The 3-10 keV and 10-20 keV $\mathrm{X}$-ray emission, generally characterizing the thermal emission, follow these impulsive changes. There are, however, several examples of impulsive increases in 10-20 keV emission which appear to be of nonthermal origin. These results are consistent with previous observations and with theoretical studies in which the chromospheric response to energetic beamed electrons is calculated, provided that the chromospheric hydrogen densities are relatively high $\left(\sim 10^{14}-10^{15} \mathrm{~cm}^{-3}\right)$.
\end{abstract}

Key words. Sun: flares - Sun: X-rays, gamma rays - Sun: chromosphere - Sun: magnetic fields

\section{Introduction}

Solar flares have been observed over the entire electromagnetic spectrum for many years, from $\gamma$-rays up to long-wavelength radio waves. Despite immense progress over the years, there are still many unsolved observational and theoretical problems in our understanding of flares, e.g. the identification of processes involved in the primary energy release in loop-top sources and the subsequent heating and chromospheric evaporation processes arising from energy that is either conducted from hot plasma contained in loops or transferred by non-thermal particle beams.

Satellite-borne hard X-ray instruments reveal the location and time variations in the flare primary energy release rate occurring in the corona. The chromospheric response to sudden impulses of energy contained in these electrons may be observed with ground-based instruments observing in chromospheric lines such as the hydrogen $\mathrm{H} \alpha$ line at $6563 \AA$. Hard $\mathrm{X}$-ray data generally have very high time resolution but until recently have been rather limited in spatial resolution; on the other hand $\mathrm{H} \alpha$ observations collected with state-of-the-art instruments on large ground-based telescopes have both high spatial and time resolution, so providing important data for investigations of the solar flares.

Theoretical models indicate that a conduction front reaches the chromosphere about 10-20 s after the onset of the hard Xray (HXR) emission (Trottet et al. 2000). Beamed non-thermal,

* Figures 12 to 17 are only available in electronic form at http: //www . aanda.org high-energy electrons reach the lower corona and upper chromosphere in $<1 \mathrm{~s}$, where they are stopped and their kinetic energy reappears as HXR bremsstrahlung and plasma heating. Model atmosphere calculations made by Heinzel (1991) predict a strong correlation of HXR and $\mathrm{H} \alpha$ emission, with a short time lag of the $\mathrm{H} \alpha$ emission compared with the HXR emission that depends on the target density. According to Heinzel's numerical models, if the heating time $\Delta t_{\mathrm{h}}$ of the chromosphere by the electron beam is about $0.1 \mathrm{~s}$, the cooling time of the chromosphere is of the order of $\Delta t_{\mathrm{c}} \sim 1 \mathrm{~s}$ and its brightness variations, observed in strong chromospheric lines, should also be of the order of $1 \mathrm{~s}$. Indeed, the HXR fluxes observed during the impulsive phase of flares are generally highly variable in time, with characteristic time scales of $<1 \mathrm{~s}$. There is hence a need for high-cadence observations in the $\mathrm{H} \alpha$ line.

Flare observations in both $\mathrm{H} \alpha$ and X-rays have been discussed by several other workers. Starting in the early 1980s, Kurokawa and his co-workers made numerous observations of solar flares using a narrow-band H $\alpha$ filter (Kurokawa et al. 1986; Kurokawa et al. (1988); Kitahara \& Kurokawa 1990). The best time resolution achieved in their observations was $\sim 1 \mathrm{~s}$. They found that the time lag between $\mathrm{H} \alpha$ and either hard X-ray or microwave emission was $<10 \mathrm{~s}$. Wang et al. (2000) made $\mathrm{H} \alpha$ observations of solar flares with a time resolution of $0.033 \mathrm{~s}$. They found that during a 7-s-long period the $\mathrm{H} \alpha$ emission of a flare kernel showed fast $(0.3-0.7 \mathrm{~s})$ fluctuations correlated with variations of the HXR flux. Also, Trottet et al. (2000) found a strong time correlation between HXR and $\mathrm{H} \alpha$ emission from a GOES X1.3 class flare on 1991 March 13. The HXR emission showed 
pulses with rise times ranging from $0.4 \mathrm{~s}$ to $1.5 \mathrm{~s}$, the crosscorrelation coefficient of the $\mathrm{H} \alpha$ and HXR fluxes being about 0.84 for a $0.5 \mathrm{~s}$ time lag of the $\mathrm{H} \alpha$ emission. In 2002, Hanaoka et al. (2004) began a programme of moderate-cadence $\mathrm{H} \alpha$ observations of solar flares, collecting images taken at line-centre as well as off-band images for velocity measurements and linear polarization measurements, with data acquisition rates of 1-2 s, $1 \mathrm{~min}$ and $45 \mathrm{~s}$. Recently, McAteer et al. (2005) presented new results of very high cadence $(0.08 \mathrm{~s}) \mathrm{H} \alpha$ blue wing observations of a C9.6 solar flare. The authors used wavelet transformations and time-distance methods to study oscillatory power along a flare ribbon. They found 3\% damped brightness oscillations with periods of 40-80 s. According to them, the measured properties of the $\mathrm{H} \alpha$ emission changes can be identified with flare-induced acoustic waves within the overlying loops.

This paper describes an investigation of $\mathrm{H} \alpha$ flare emission with observations having very high time cadence (between 0.075 and $0.04 \mathrm{~s}$, i.e. about 13 to 25 images $\mathrm{s}^{-1}$ ) using the Large Coronagraph $(L C)$, Multi-Channel Subtractive Double Pass spectrograph $(M S D P)$ and fast CCD cameras, part of the Solar Eclipse Coronal Imaging System (SECIS), at the Białkow Observatory of the University of Wrocław, Poland. It discusses the correlation of these data with hard X-ray emission recorded with the Reuven Ramaty High Energy Solar Spectroscopic Imager (RHESSI). Unlike previous work, the observations made with the MSDP spectrograph allow us to make a comparison of the emission observed simultaneously at several specifiable wavelengths across the $\mathrm{H} \alpha$ line profile, including the line centre and blue and red wings. The observations are timetagged with atomic clock information. The $\mathrm{H} \alpha$ data are compared with RHESSI data with 4-s time resolution, determined by the period of the spacecraft rotation, and with data demodulated with recently developed software, giving a time resolution of $0.25 \mathrm{~s}$. From data collected over two observing seasons (2003 and 2004), we selected four flares observed on two days for detailed discussion. Some preliminary results of this work have already been presented (Radziszewski et al. 2005; Radziszewski et al. 2006). More recent observations of a number of other flares will be described in a future work (Paper II).

\section{Instrumentation and data reduction}

The $\mathrm{H} \alpha$ spectral-imaging data were collected with the $L C$ equipped with the MSDP spectrograph and SECIS CCD cameras at the Białkow Observatory of the Wrocław University. The $L C$ has a $53 \mathrm{~cm}$ diameter main objective, its effective focal length is $1450 \mathrm{~cm}$, and spatial resolution, normally limited by seeing conditions, about 1 arcsec or better. The $M S D P$ spectrograph has a rectangular entrance window, which covers an equivalent area of $325 \times 41 \operatorname{arcsec}^{2}$ on the Sun (Mein 1991; Rompolt et al. 1994). The spectrograph has a nine-channel "prism-box", enabling restoration of the $\mathrm{H} \alpha$ line profiles in the range $\pm 1.2 \AA$ from the line centre using the emission measured in nine wavelengths separated by $0.4 \AA$. The spectra-images created by the MSDP spectrograph were recorded with one of a pair of fast CCD cameras of SECIS. The SECIS cameras, manufactured by EEV (UK), have a $512 \times 512$ pixels $(\mathrm{px})$ image format, and images are acquired with frequencies of up to 70 images $\mathrm{s}^{-1}$. The data are digitized to effectively 10 bits, so that the dynamic range is approximately 1000:1. SECIS was originally designed and used by British-Polish expeditions for investigation of fast intensity changes in solar coronal features during the total solar eclipses in 1999, 2001, and 2006 (Phillips et al. 2000; Rudawy et al. 2004). The image scale for the present work was such that the camera pixel size was close to 1 arcsec. The SECIS camera software allows the collection of 10000 images for each set of observations, but the time cadence can be adjusted according to the light intensity. For the observations discussed here, the time cadence selected was between $0.04 \mathrm{~s}\left(25\right.$ images $\left.\mathrm{s}^{-1}\right)$ and $0.075 \mathrm{~s}$ $\left(\sim 13\right.$ images $\left.\mathrm{s}^{-1}\right)$.

The raw MSDP/SECIS data were photometrically corrected in a standard way, using flat-field and dark current images. Additionally the data taken on 2003 July 16 were corrected for Newton's fringes, accidentally superimposed on the images. As a result of the numerical reduction of the collected spectra-images, for each flare we obtained a series of 10000 sets consisting of thirteen two-dimensional, quasi-monochromatic (band-width $=0.06 \AA$ ) images, separated by $0.2 \AA$, at wavelengths $\pm 1.2 \AA$ from the $\mathrm{H} \alpha$ line centre. To show all the structures visible in entire $\mathrm{H} \alpha$ line profile, we constructed compound images consisting of all quasi-monochromatic images taken across the line profile. Using these data we were also able to restore the $\mathrm{H} \alpha$ line profiles for each pixel in the field of view. The collected data allow us to investigate not only the local time changes of the $\mathrm{H} \alpha$ emission in the laboratory (zero motion) and Doppler-shifted (associated with the emitting material) wavelength systems, but also to measure true velocities and emission profiles of the emitting material.

A permanent characteristic feature of the MSDP quasimonochromatic images is a series of parallel strips of slightly different brightness extending along the whole image, caused by uncertainties of the spline interpolation of the observed profiles. In order to compensate for this (and the variation of atmospheric transmittance), each measured signal was normalized using the average emission of an adjacent region of the quiet chromosphere lying along the same brightness strip.

Despite the massive nature of the $L C$, its 10-meter-long main frame is sensitive to wind gusts. During very windy days the solar image moved across the camera detector by up to about 10 arcsecs, showing slow, large-excursion drifts and small, noise-like changes. For this reason, on such days the $L C$ was replaced by a Horizontal Telescope with a compact Jensch-type coelostat, the main objective of which has an aperture of $15 \mathrm{~cm}$ and focal length $5 \mathrm{~m}$. When the $L C$ was used during less windy days, the wind-induced residual motions of the field of view were corrected by shifting all images to a common, reference position by means of 2-D correlations of well defined chromospheric structures with an accuracy of about $1 \mathrm{px}$. It was our intention to improve the co-alignment of the images by evaluating the residual shifts to less than $0.25 \mathrm{px}$. To do so, we attempted to evaluate such sub-pixel shifts using a 2-D correlation of the chromospheric structures at the reference and actual images, both rebinned to 10-times-smaller pixels. Unfortunately, after some numerical experiments we found the reliable improvement of the pointing below 1 px was impossible, while the seeing, acting in an unpredictable, stochastic manner, caused some local deformations of the images, visible as changes of the shape and brightness of the observed structures.

As a result, for each $\mathrm{H} \alpha$ source we evaluated only the mean emission calculated inside a rectangular area big enough to encompass the source during a particular sequence of images. The averaged intensities obtained were plotted as a light curve for each $\mathrm{H} \alpha$ emission source. The disadvantage of this method is that the emission of the source is averaged with some emission of the surrounding chromosphere, significantly lowering the fractional variations of the signal. An example of the evolution of high-cadence $\mathrm{H} \alpha$ line spectra obtained with MSDP-SECIS 


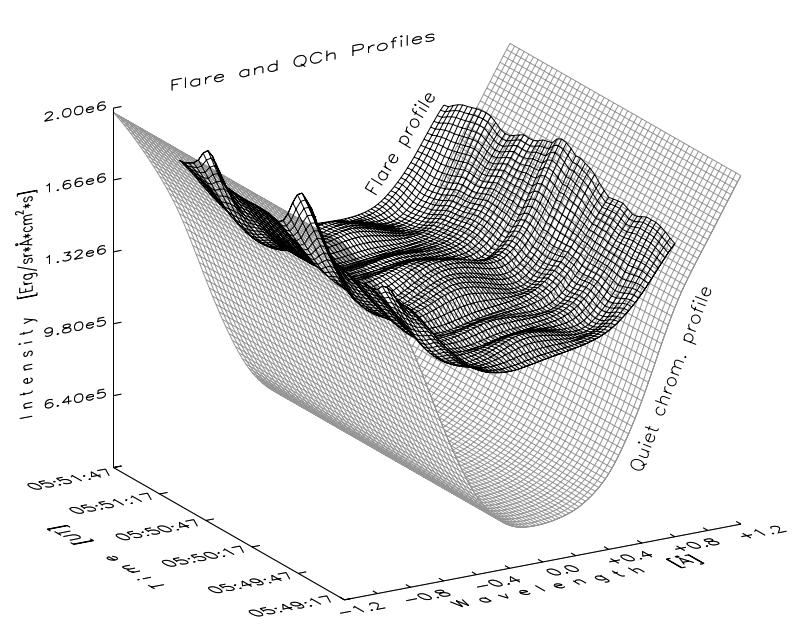

Fig. 1. An example of the high-cadence spectral observations collected with the $L C, M S D P$ spectrograph and SECIS system (see main text for details). The graph shows the time evolution of the $\mathrm{H} \alpha$ line profiles emitted by the B9.1 class solar flare on 2004 April 23. The time resolution of the observations was $0.075 \mathrm{~s}$, and the bandwidth of the profiles was limited to $\pm 0.8 \AA$. For comparison the mean profile of the nearby quiet solar chromosphere in the range $\pm 1.2 \AA$ is shown.

system is shown in Fig. 1. Each spectrum in the series is measured for the rectangular area covering a bright kernel of the B9.1 class solar flare on 2004 April 23 (marked as K4 in Fig. 2). For comparison, the mean profile of the nearby solar quiet chromosphere is also shown.

Our observations are the first ever obtained of a long (several-minute) series of $\mathrm{H} \alpha$ line spectra and images of flaring kernels with time resolution as fine as $0.04 \mathrm{~s}$. Such high-timeresolution spectra allow the investigation of time variations of the flare emission at different parts of the $\mathrm{H} \alpha$ profile simultaneously, and so can assist in modelling the chromospheric response to electron beams during the flare impulsive phase (e.g. Heinzel 2003).

The $\mathrm{H} \alpha$ light curves were compared and correlated with $\mathrm{X}$-ray light curves recorded with RHESSI. Imaging of X-ray flares with RHESSI is accomplished through the use of rotating modulation collimators (Lin et al. 2002; Hurford et al. 2002) located in front of the detectors. Each collimator consists of a pair of grids made up of parallel slats and slits. As the spacecraft rotates (with a period of about $4 \mathrm{~s}$ ), the flare X-ray signal is modulated in time. Grids with smallest pitch modulate the incoming flux fastest and their output leads to images with the finest spatial resolution (2.3 arcsec). Image reconstruction from the modulated light curves is via various algorithms such as "back projection" in software packages written by the RHESSI team. At increasing photon count rates during flares, sets of attenuators are inserted in front of the detectors to avoid detector saturation; the attenuator states used are A0 (no attenuator), A1 (thin attenuators) and A3 (thick and thin attenuators together). Attenuator changes occurred during two of the four flares studied here, but only one (see Fig. 9) affects the analysis of the H $\alpha$ and RHESSI data.

$\mathrm{X}$-ray light curves from RHESSI are generally limited to a time resolution of $\sim 4 \mathrm{~s}$, this being the spacecraft spin period, but it is possible to get finer time resolution by "demodulating" the output. A test version of a demodulation program was written in Interactive Data Language (IDL) and made available to us by G. J. Hurford (Hurford 2004). The program takes the modulated output from the detectors and performs a demodulation with a time resolution selected by the user, which in the present version of the program is between 0.05 and $0.25 \mathrm{~s}$. A sufficiently large count rate $(\geq 150$ counts summed over the detectors used per finest time interval of interest) is required. No particular detector combinations are needed by the program, but for energies $<8 \mathrm{keV}$ we used data from all detectors except detectors 2 and 7 (which have relatively poor spectral resolution and high energy thresholds); for energies $>8 \mathrm{keV}$ we used data from all detectors except detector 2 . In our analysis we used the demodulator program to form RHESSI X-ray light curves with $0.25 \mathrm{~s}$ time resolution. The energy range of RHESSI is $3 \mathrm{keV}$ to $17 \mathrm{MeV}$, so includes both soft ( $\lesssim 12 \mathrm{keV})$ and hard $(\gtrsim 12 \mathrm{keV}) \mathrm{X}$-ray energies as well as $\gamma$-rays. For comparison with our $\mathrm{H} \alpha$ data, we integrated RHESSI photon counts in the ranges $3-10 \mathrm{keV}, 10-20 \mathrm{keV}$ and $20-50 \mathrm{keV}$ (though the photon count rates at higher energies were sometimes very small).

\section{Description of the observed flares}

Four flares were chosen for detailed analysis, one on the disk, observed on 2003 July 16, and three near the west limb, observed on 2004 April 23 (see Table 1). Figure 2 shows $\mathrm{H} \alpha$ line centre images of bright emission sources, labelled K1-K8, and of quiet chromosphere reference regions, labelled Q, which were used to compensate the uncertainties of the photometry caused by the variation of the atmospheric transmittance and striped structure of the MSDP images, described in Sect. 2.

The 2003 July 16 flare was a C1.2 GOES class solar flare in active region NOAA 10410 at $\mathrm{S} 12^{\circ} \mathrm{E} 38^{\circ}$. The active region appeared close to the east solar limb on July 12 and grew gradually over the next four days, maintaining a $\beta$ magnetic class. Solar flares were first observed in the region on July 16: at 12:15 UT (C1.3 class flare), at 16:01 UT (C1.2, this flare was observed by us, see Fig. 3) and at 16:28 UT (C2.9 class flare). By this time, its sunspot group consisted of a single large leading spot and a following group of scattered small spots. Enhanced $\mathrm{H} \alpha$ emission was concentrated to the west and to north of the main sunspot, along the magnetic neutral line. We made high-cadence $\mathrm{H} \alpha$ observations of the active region on July 16 between 15:57:45 UT and 16:06:05 UT with a time resolution of $0.05 \mathrm{~s}\left(20\right.$ images $\left.\mathrm{s}^{-1}\right)$. This flare was also observed by RHESSI. For this active region we also made some low time resolution context MSDP observations before and after the flare.

On 2004 April 23, we observed three solar flares in active region NOAA 10597. This active region was first observed on April 21 as a small, bipolar sunspot group of $\beta$ magnetic class. It grew slightly during the next two days, but did not produce any flare. On April 23 the active region was located at $\mathrm{S} 06^{\circ} \mathrm{W} 83^{\circ}$, very close to the Sun's west limb. It had become very active by then, producing $18 \mathrm{C}$-class and $2 \mathrm{M}$-class flares during a single day. Our first set of $\mathrm{H} \alpha$ observations was taken on April 23 between 05:49:17 UT and 06:01:48 UT, during a B9.1 GOES class flare which started at 05:47 UT, peaked at 05:50 UT, and ended at 05:52 UT (see Fig. 4). Our second set of $\mathrm{H} \alpha$ observations was taken between 09:28:50 UT and 09:41:19 UT, during a C4.4 GOES class flare which started at 09:25 UT, peaked at 09:30 UT, and ended at 09:32 UT. Both the H $\alpha$ flares were compact with bright kernels. Unfortunately, for both these flares, the emission sources were partially obscured by an elongated structure perpendicular to the solar limb, possibly the lower part of a loop structure, filled by absorbing material (see Figs. 4 and 5). During the 09:30 flare a surge was ejected along this structure. In the $\mathrm{H} \alpha$ compound images (see Fig. 5, right column) the flaring 
Table 1. High cadence H $\alpha$ observations made on 2003 July 16 and 2004 April 23 at the Białkow Observatory.

\begin{tabular}{|c|c|c|c|c|c|c|c|c|}
\hline Data & $\begin{array}{l}\text { NOAA } \\
\text { region }\end{array}$ & $\begin{array}{l}\text { GOES } \\
\text { class }\end{array}$ & $\begin{array}{c}\text { GOES Flare } \\
\text { start-peak-end [UT] }\end{array}$ & $\begin{array}{l}\mathrm{H} \alpha \text { observations } \\
\text { start-end [UT] }\end{array}$ & $\begin{array}{l}\text { Number } \\
\text { of images }\end{array}$ & $\begin{array}{c}\text { Cadence } \\
{[\mathrm{s}]}\end{array}$ & Białkow Obs. & RHESSI \\
\hline $\begin{array}{c}\text { July } 16 \\
2003\end{array}$ & $\begin{array}{c}10410 \\
(\mathrm{~S} 12 \mathrm{E} 38)\end{array}$ & C1.2 & $16: 01-16: 10-16: 24$ & $\begin{array}{l}15: 37: 31-15: 57: 45 \\
15: 57: 45-16: 06: 05 \\
16: 06: 43-16: 52: 33\end{array}$ & $\begin{array}{c}3 \\
10000 \\
75\end{array}$ & $\begin{array}{c}\sim 50 \\
0.050 \\
\sim 50\end{array}$ & $\begin{array}{l}\text { LC-MSDP (context) } \\
\text { LC-MSDP-SECIS } \\
\text { LC-MSDP (context) }\end{array}$ & yes \\
\hline $\begin{array}{c}\text { April } 23 \\
2004\end{array}$ & $\begin{array}{c}10597 \\
(\mathrm{~S} 06 \mathrm{~W} 83)\end{array}$ & $\begin{array}{l}\text { B9.1 } \\
\text { C4.4 } \\
\text { M1.5 }\end{array}$ & $\begin{array}{l}05: 47-05: 50-05: 52 \\
09: 25-09: 30-09: 32 \\
11: 41-11: 50-11: 52\end{array}$ & $\begin{array}{l}05: 49: 17-06: 01: 48 \\
09: 28: 50-09: 41: 19 \\
11: 49: 38-11: 56: 18\end{array}$ & $\begin{array}{l}10000 \\
10000 \\
10000\end{array}$ & $\begin{array}{l}0.075 \\
0.075 \\
0.040\end{array}$ & LC-MSDP-SECIS & $\begin{array}{c}\text { yes } \\
\text { yes } \\
\text { partial }\end{array}$ \\
\hline
\end{tabular}
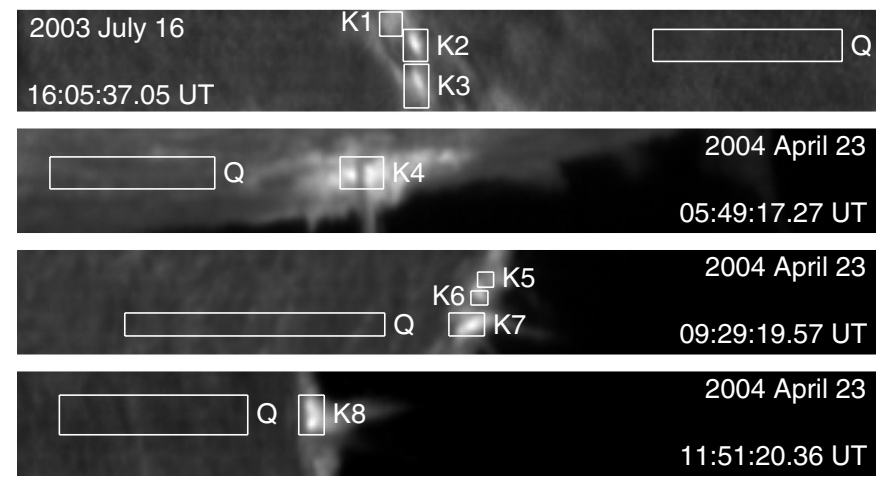

Fig. 2. $\mathrm{H} \alpha$ line centre images of the solar disk flare on 2003 July 16 and the three limb flares on 2004 April 23 observed with the LC-MSDPSECIS system at the Białkow Observatory. The $\mathrm{H} \alpha$ emission sources referred to in the text are marked K1-K8, while relevant reference quietchromosphere regions are marked Q. Flare characteristics are given in Table 1.

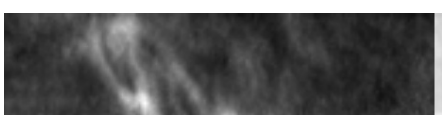

16:02:00.00 UT

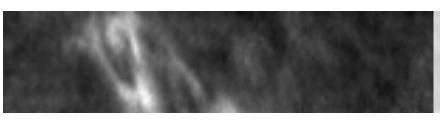

16:03:30.00 UT

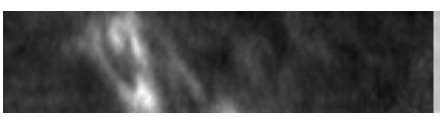

16:03:43.50 UT
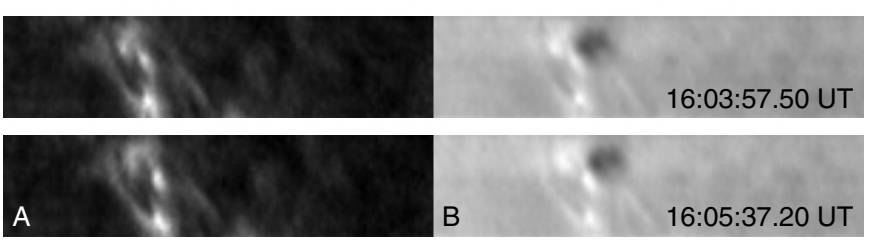

Fig. 3. Left column A): $\mathrm{H} \alpha$ line centre images of the flare (kernels K1, $\mathrm{K} 2$ and K3) in the active region NOAA 10410 on 2003 July 16. Right column B): compound images (sum of eleven quasi-monochromatic images taken in range $\pm 1 \AA$, including $\mathrm{H} \alpha$ line centre) of the same flare. The images were taken during the rise of the flare, including the impulsive phase, with the LC-MSDP-SECIS system at Białkow Observatory.

kernels marked K5 and K6 are evident, but in the $\mathrm{H} \alpha$ line centre images (left column) the K5 kernel is not very conspicuous. The time resolution of the $\mathrm{H} \alpha$ data obtained during these flares was $0.075 \mathrm{~s}$ (about 13 images s${ }^{-1}$ ). The spatial resolution of the images was limited by seeing to about 1 arcsec. Both events were observed by RHESSI (no other flares occurred on the disk, as was checked from GOES-12 SXI images).

A final set of $\mathrm{H} \alpha$ observations on 2004 April 23 was taken between 11:49:38 UT and 11:56:18 UT, during the M1.5 GOES class solar flare in the same active region. This flare, the largest
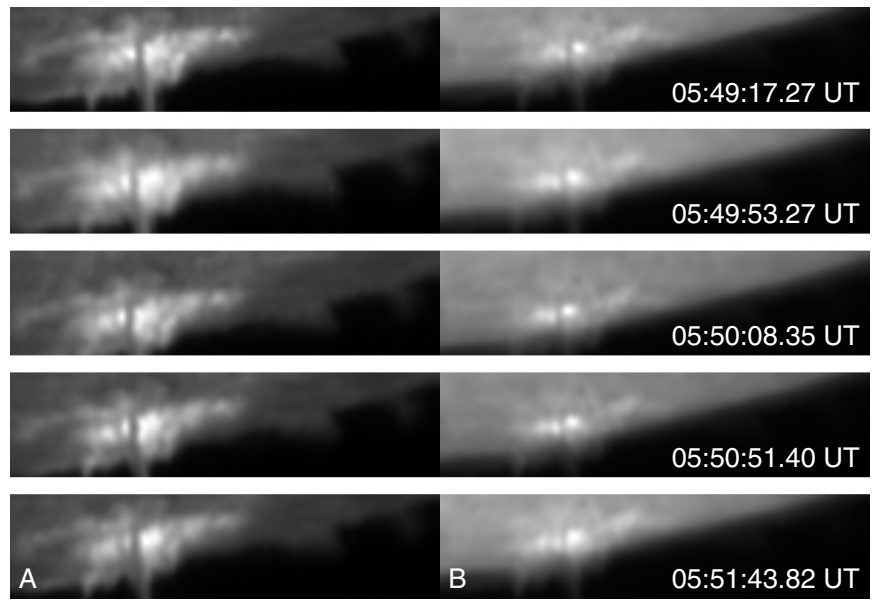

Fig. 4. A) $\mathrm{H} \alpha$ line centre images, B) compound images, for the B9.1 flare (kernel K4) observed on the west limb in active region NOAA 10597 on 2004 April 23. The images were taken during the flare's impulsive phase. The K4 kernel was partially obscured by a dark structure perpendicular to the limb.
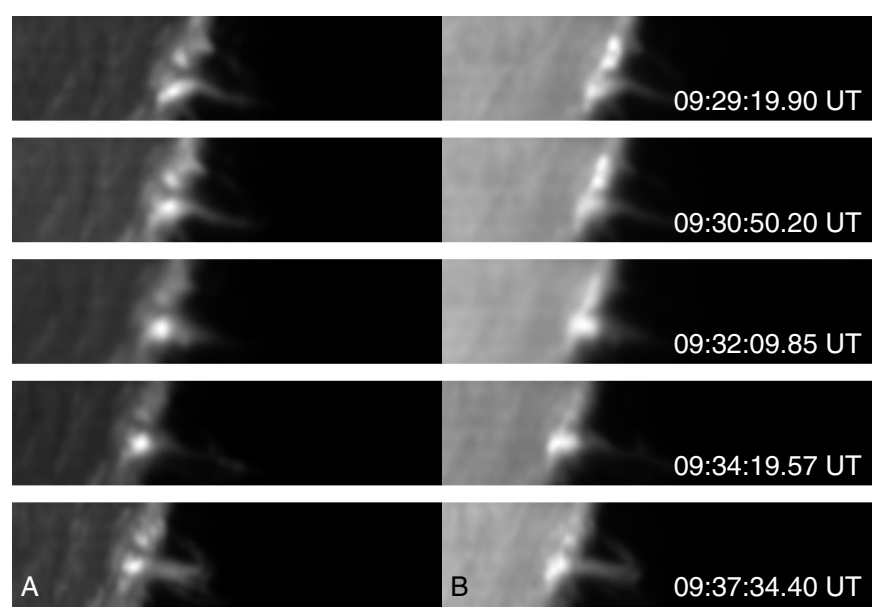

Fig. 5. A) $\mathrm{H} \alpha$ line centre images, B) compound images, for the $\mathrm{C} 4.4$ flare (flaring kernels K5, K6 and emission source K7) observed on the west limb in active region NOAA 10597 on 2004 April 23.

of the four described here, started at 11:41 UT, peaked at 11:50 UT and ended at 11:52 UT. In H $\alpha$ the flare appeared as a bright, compact loop located near the lower part of an elongated structure perpendicular to the limb, filled by rising material (see Fig. 6). The footpoints of the bright low loop, marked as K8 in Fig. 2, are co-spatial with the two structures marked as K5 and K6 during the 09:30 UT flare and are better seen in the compound images presented in Fig. 6, right column. Unfortunately, there is very little coverage (approximately $100 \mathrm{~s}$ ) of this flare by RHESSI observations as RHESSI had just emerged from 

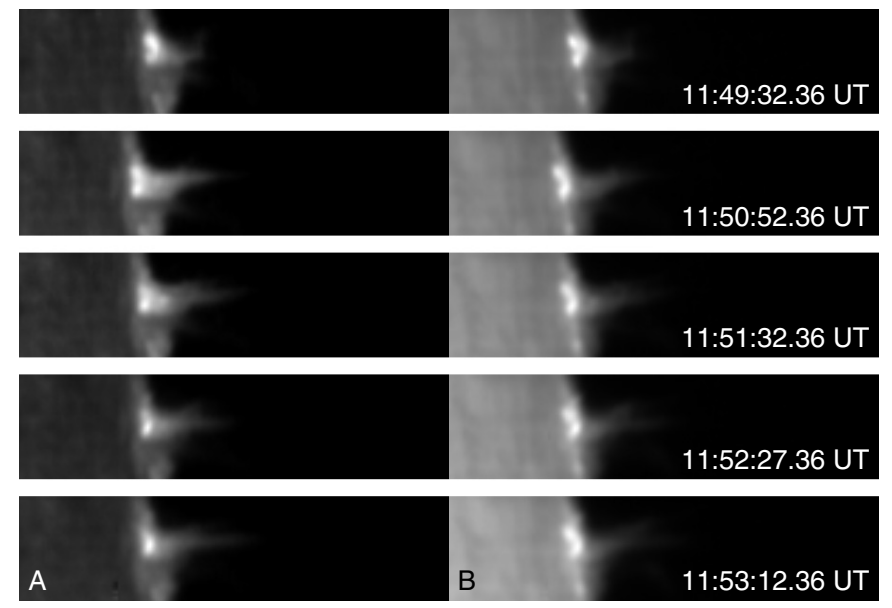

Fig. 6. A) $\mathrm{H} \alpha$ line centre images, B) compound images, of the M1.5 flare (kernel K8) on the west limb in active region NOAA 10597 on 2004 April 23.

spacecraft night at the start of our observations and then immediately entered the South Atlantic Anomaly.

\section{Results}

The $\mathrm{H} \alpha$ and RHESSI X-ray light curves of the four solar flares are shown in Figs. 7-11. Further plots, showing more detail, are given in Figs. 12-17 in on-line material associated with this paper. For each $\mathrm{H} \alpha$ emission source $(\mathrm{K} 1-\mathrm{K} 8)$ the $\mathrm{H} \alpha$ data are shown with integration periods of $4 \mathrm{~s}$ and $0.25 \mathrm{~s}$ as well as with the full time resolution $(0.04 \mathrm{~s}-0.075 \mathrm{~s})$. To reduce the noise in the higher-cadence light curves, the data were smoothed with a box-car filter to $1 \mathrm{~s}$ (shown as dark lines); the unsmoothed data are also plotted in each case (light grey lines). The RHESSI light curves are shown with integration times of $4 \mathrm{~s}$ (no demodulation: top panels) and $0.25 \mathrm{~s}$ (de-modulated data: middle panels) for three energy bands (3-10 keV; 10-20 keV and 20-50 keV). The $0.25 \mathrm{~s}$ integrated data, smoothed with a $2 \mathrm{~s}$ box car filter, were shown as dark lines; as with the $\mathrm{H} \alpha$ data, the unsmoothed data are plotted with light grey lines. The RHESSI fluxes are plotted logarithmically, the $\mathrm{H} \alpha$ fluxes linearly, the scales being relative in each case. For X-ray energies less than $20 \mathrm{keV}$, discernible variations in the RHESSI light curves may be considered real and of solar origin as the count rates are very high. For the highest energy range $(20-50 \mathrm{keV})$ the count rates are lower, and the Poisson uncertainties in the rates are appreciable. We estimated the latter (from an algorithm supplied to us by R. A. Schwartz of the RHESSI team) and illustrate their magnitude by the error bars shown in each of the four figures for the largest count rates.

\subsection{C1.2 flare on 2003 July 16}

The 3-10 keV and 10-20 keV X-ray light curves (4 s integration) for this flare show a gradual rise over the period 16:02:30-16:05:30 UT, with the 10-20 keV showing a maximum at 16:04:16 UT These gradual changes most likely follow the flare's thermal emission but the 10-20 keV energy channel also includes many short-lived intensity increases that appear to be of nonthermal origin. The $20-50 \mathrm{keV}$ X-ray emission has an impulsive main peak at 16:03:54 UT, of high statistical significance, with a $\sim 20 \mathrm{~s}$ rise and $\sim 10 \mathrm{~s}$ fall (Figs. 7 and 11). It is still discernible in the demodulated data $(0.25 \mathrm{~s}$ integration), with the possibility of a small peak corresponding to it on the rise in the 10-20 keV X-ray emission, though this has low significance. The impulsive peak has a clear counterpart in the $\mathrm{H} \alpha$ emission from the $\mathrm{K} 1 \mathrm{kernel}$. For the $\mathrm{H} \alpha$ line centre and wing emission, the K1 peak occurs at 16:03:57 UT, so a $\sim 3$ s time lag between the hard X-ray and $\mathrm{H} \alpha$ line emission is indicated (Fig. 11). Hardly any response to the hard X-ray peak is indicated in the $\mathrm{K} 2$ or $\mathrm{K} 3$ kernel $\mathrm{H} \alpha$ emission with the possible exception of a slight decrease in $\mathrm{H} \alpha$ red wing emission at 16:04:10 UT, just after the $20-50 \mathrm{keV}$ X-ray peak. The maximum of the thermal emission in the $10-20 \mathrm{keV}$ X-ray range occurs $\sim 25 \mathrm{~s}$ or more after the $20-50 \mathrm{keV}$ and $\mathrm{H} \alpha \mathrm{K} 1$ peaks. At the softer energy of the GOES 1-8 $\AA$ channel, there is a peak at the still later time of 16:10 UT, some 6 minutes after the 20-50 keV impulsive peak. We note that the $\mathrm{K} 1$ kernel is relatively faint in $\mathrm{H} \alpha$ compared with the K2 and K3 kernels (Figs. 2 and 3) but has the clearest indication of the flare impulsive phase shown at high-energy (20-50 keV) X-rays.

In addition, we observed differences in the timings in the first increases of the $\mathrm{H} \alpha$ emission from the three kernels of this flare, and even between different parts of the $\mathrm{H} \alpha$ line profile from the same kernel. Of particular note is a 26-s difference in the start time of the $\mathrm{H} \alpha$ red and blue wing emission in the K1 kernel (see Figs. 7, 11, and 12 (on-line material)). There is also a 38-s time difference between the time of increase in the $\mathrm{K} 1$ and $\mathrm{K} 3$ kernels in the $\mathrm{H} \alpha$ blue wing and a 22-s difference in the red wing (Figs. 7 and 12-14 (on-line material)). Some variations in emission occurring between 16:04:10 and 16:04:50 UT are more marked in the $\mathrm{H} \alpha$ red wing and line centre emission than in the $\mathrm{H} \alpha$ blue wing.

\subsection{B9.1 flare on 2004 April 23}

The 3-10 keV and 10-20 keV X-ray light curves show a 30-s rise to a maximum at 05:50:26 UT, followed by a more gradual fall. The peak is also evident at the same time in higher (20-50 keV) energy emission, followed by a sharper fall, still just discernible in the demodulated $(0.25 \mathrm{~s})$ data in the $3-10$ and 10-20 keV ranges. The demodulated data at these energies show an earlier maximum, at 05:50:15 UT. There is an $\mathrm{H} \alpha$ response to the flare maximum at 05:50:29 UT in kernel K4 at the $\mathrm{H} \alpha$ line centre but not in the $\mathrm{H} \alpha$ line wings (Figs. 8, 11, and 15 (online material)). The maximum in the $\mathrm{K} 4 \mathrm{H} \alpha$ line centre emission is thus $\sim 2 \mathrm{~s}$ later than the $20-50 \mathrm{keV} \mathrm{X}$-ray peak. Only a very small time lag of $\mathrm{H} \alpha$ emission is therefore indicated, identical to that for lower X-ray energies (3-10 keV, 10-20 keV) for which one normally expects thermal emission to be dominant and so a later maximum time which is not observed. Our interpretation is that the impulsive, nonthermal peak at 05:50:26 UT apparent at high X-ray energies also extends to soft X-ray energies, as has been noted several times for other flares (Hudson et al. 1994; Lin et al. 2001; Mrozek \& Tomczak 2004).

\subsection{C4.4 flare on 2004 April 23}

RHESSI observations of this flare extend from 09:29 UT to 09:38 UT, though there is an attenuator change (from A1 to A0) at 09:33 UT - the data are consequently noisier before this time owing to higher count rates in the A0 state. The $20-50 \mathrm{keV} X$-ray emission shows a strong peak at 09:29:48 UT, still visible in the demodulated data, which precedes by a few s the corresponding maximum in the 3-10 keV and 10-20 keV light curves (Fig. 9). Two small emission kernels (K5 and K6) and a larger source 


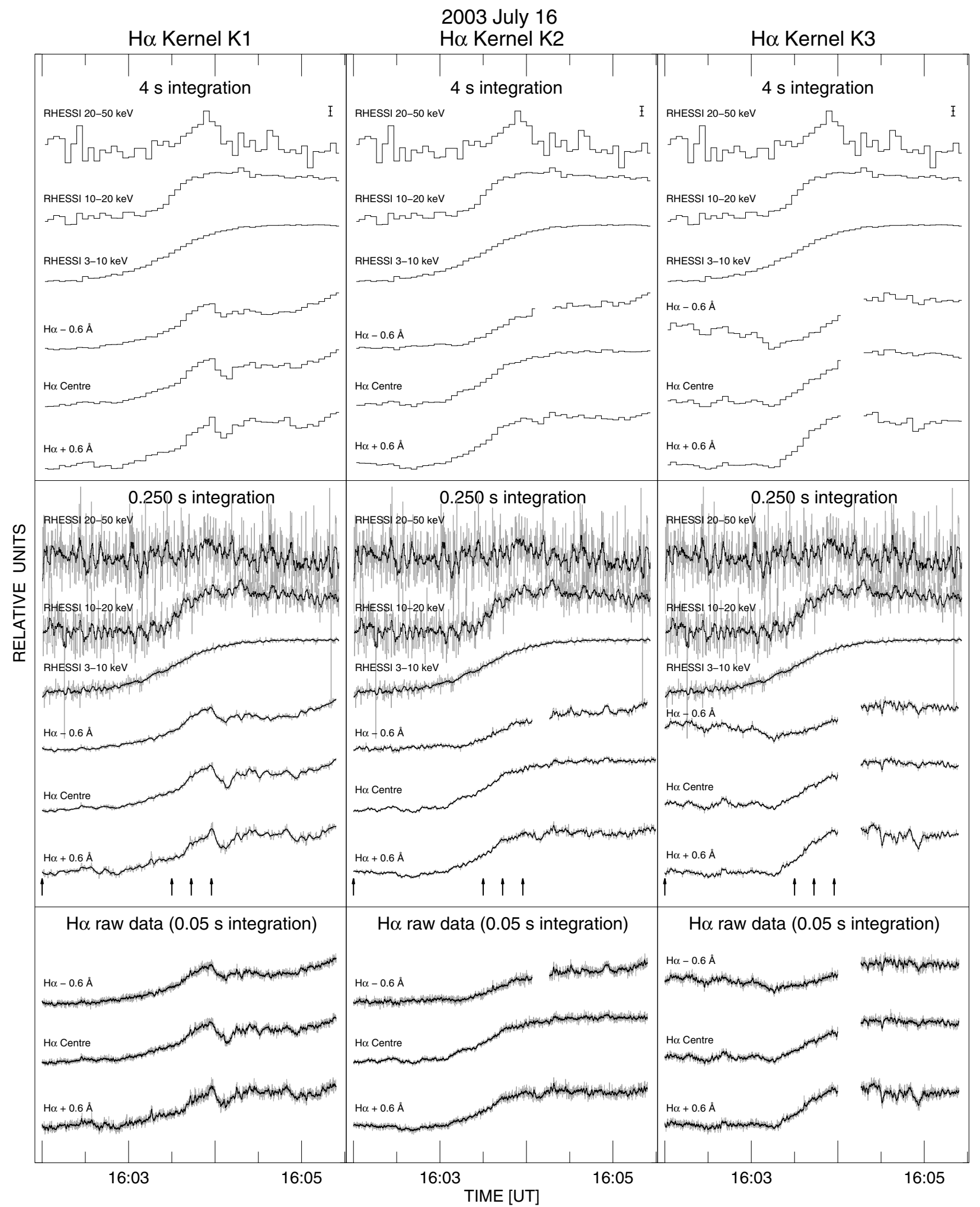

Fig. 7. The time-series of the RHESSI X-ray and LC-MSDP-SECIS $\mathrm{H} \alpha$ fluxes of the flaring kernels $\mathrm{K} 1, \mathrm{~K} 2, \mathrm{~K} 3$ (shown in the three columns of the figure) recorded during the C1.2 flare in NOAA active region 10410 on 2003 July 16 . The H $\alpha$ data are shown for line centre and $\pm 0.6 \AA$ using a linear scale. The integration times are equal to $4 \mathrm{~s}, 0.25 \mathrm{~s}$, and $0.05 \mathrm{~s}$ in top, middle and bottom panels respectively. The data were smoothed using $1 \mathrm{~s}$ box-car filter (shown as dark lines; the unsmoothed data are shown as grey lines). The RHESSI data are photon count rates and are plotted logarithmically with $4 \mathrm{~s}$ integration times in the top panel and with $0.25 \mathrm{~s}$ integration times (i.e. with de-modulation) smoothed with $2 \mathrm{~s}$ box-car in the middle panel. The error bars indicate Poisson uncertainties in the RHESSI 20-50 keV count rates; the uncertainties for 3-10 keV and $10-20 \mathrm{keV}$ count rates are too small to be shown. The vertical scales are arbitrary. The vertical arrows in the middle panels indicate the times of the four flare images in Fig. 3. 


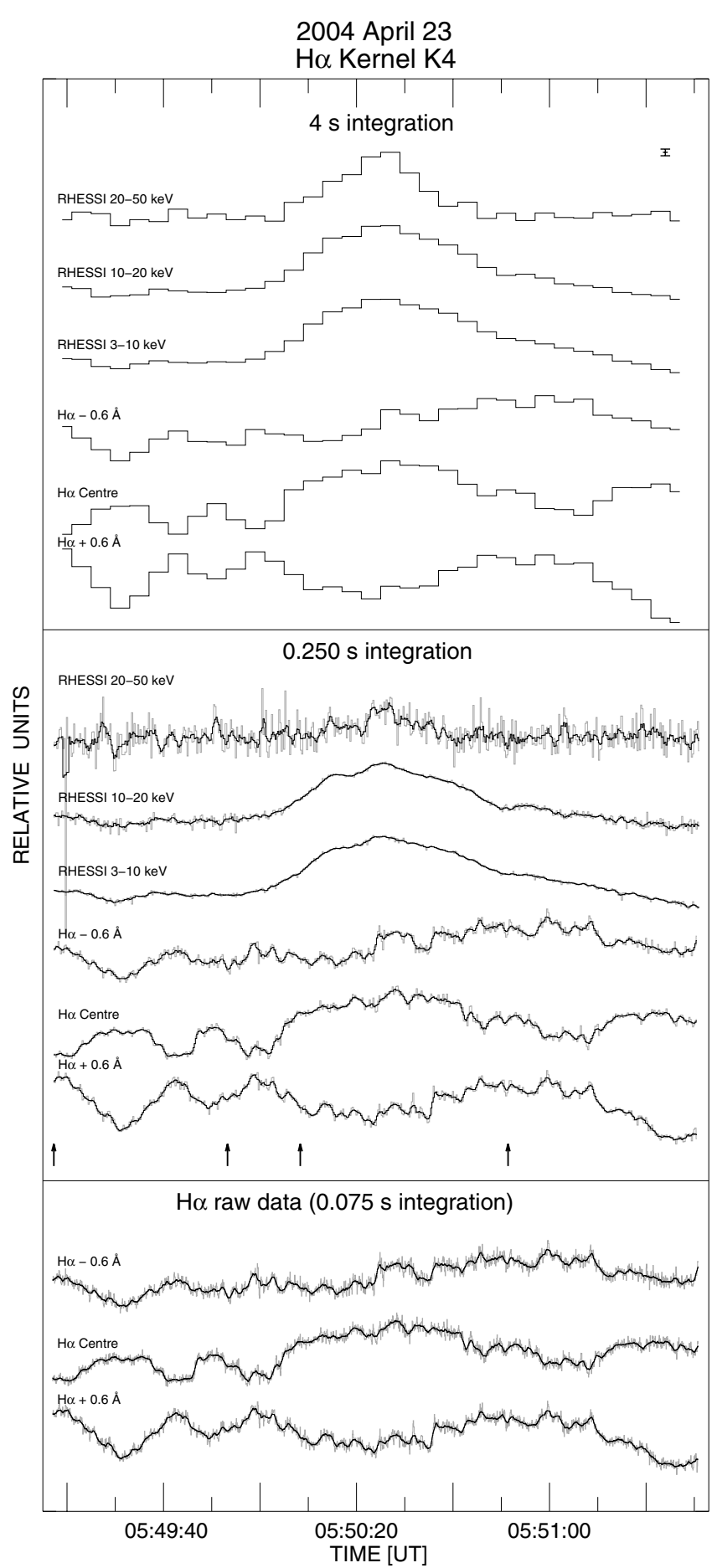

Fig. 8. The time-series of the RHESSI X-ray and LC-MSDP-SECIS $\mathrm{H} \alpha$ fluxes of the K4 flaring kernel recorded during the B9.1 flare in NOAA 10597 active region on 2004 April 23. The observations are arranged as in Fig. 7. The raw $\mathrm{H} \alpha$ data were taken with 0.075 s time resolution. The error bars indicate the uncertainties in the $20-50 \mathrm{keV}$ count rates. The vertical arrows indicate the times of the flare images in Fig. 4.

(K7) are observed in the flare $\mathrm{H} \alpha$ images (Fig. 5). Kernels K5 and K6 are possibly at the footpoints of a compact loop, while $\mathrm{K} 7$ is probably at the base of a larger, loop-like feature partly over the limb. Most likely, for this limb flare, the $\mathrm{H} \alpha$ line centre emission from all emission sources are affected by absorbing chromospheric material. Thus, for kernel K5 there is a dip in emission at the $\mathrm{H} \alpha$ line centre at the time of the X-ray maximum, but the $\mathrm{H} \alpha$ emission in the red and blue wings has a maximum at 09:30:05 UT, $\sim 17 \mathrm{~s}$ later than the peak of the 20$50 \mathrm{keV}$ X-ray emission (Figs. 9, 11 and 16 (on-line material)). The dip in the $\mathrm{H} \alpha$ line centre light curve incidentally confirms the findings of Kurokawa (1983) that $\mathrm{H} \alpha$ line centre emission is more susceptible to absorption by chromospheric material than that in the line wings. Within the $\mathrm{K} 5 \mathrm{H} \alpha$ line centre emission dip is a small but significant peak at 09:29:54 UT, or $\sim 6 \mathrm{~s}$ later than the $20-50 \mathrm{keV} X$-ray peak. There is a similarly small peak in the $\mathrm{K} 6 \mathrm{H} \alpha$ line centre light curves at this time, followed by a further maximum at 09:30:20 UT.

For the emission source $\mathrm{K} 7$, there is little in the $\mathrm{H} \alpha$ emission that can be easily related to the X-ray light curves. The increase of the 20-50 keV X-ray emission at times after 09:33:48 UT is coincident with an $\mathrm{H} \alpha$ surge.

\subsection{M1.5 flare on 2004 April 23}

Kernel K8 was located in the same small magnetic loop that is presumed to have footpoints at K5 and K6 in the 09:30 UT flare, two hours earlier (see Fig. 6). Unfortunately, RHESSI data are only available after the flare maximum, and there are no features apparent in the X-ray light curves that are recognizable in the $\mathrm{H} \alpha$ emission for the period of overlap (11:52:16-11:53:56 UT: (Fig. 10)).

\section{Discussion and conclusions}

In this study, the time variations in the $\mathrm{H} \alpha$ flare emission as observed by our MSDP/SECIS instrumentation have been compared with hard X-ray emission as observed by RHESSI. Of particular interest are short-lived, impulsive variations in both the $\mathrm{H} \alpha$ and hard X-ray emission, since a correlation would indicate the possibility of excitation of the $\mathrm{H} \alpha$ emission in bright flare kernels by electron beams or by conduction fronts proceeding along flare loop structures during the flare impulsive stage. We have identified three possible candidate features in the $\mathrm{H} \alpha$ light curves for which there are close correlations with impulsive hard $\mathrm{X}$-ray peaks. These are shown in Fig. 11.

The clearest case is the correlation of RHESSI $20-50 \mathrm{keV}$ X-ray emission and $\mathrm{H} \alpha$ emission from the $\mathrm{K} 1$ kernel during the 2003 July 16 (16:04 UT) flare. The $\mathrm{H} \alpha$ emission features of this flare, located on the disk, do not suffer from obscuration by absorbing features. The peak in $20-50 \mathrm{keV}$ X-ray emission at 16:03:54 UT closely corresponds to a peak in the light curves of the $\mathrm{H} \alpha$ emission at line centre and in the blue and red wings some $\sim 3 \mathrm{~s}$ later. The $\mathrm{K} 1 \mathrm{kernel}$ is comparatively faint in $\mathrm{H} \alpha$ compared with the K2 and K3 kernels for which there is either no or very little response to the impulsive burst seen in hard X-rays.

Another case is the correlation of RHESSI 20-50 keV X-ray emission with $\mathrm{H} \alpha$ line centre emission from the K4 flare during the limb flare of 2004 April 23 (05:50:26 UT). There is apparently no response in the $\mathrm{H} \alpha$ line wing emission. The $\mathrm{K} 4$ emission is affected by absorbing chromospheric material, but notwithstanding this there appears to be good correlation of the $\mathrm{H} \alpha$ line centre feature some $\sim 2 \mathrm{~s}$ after the $20-50 \mathrm{keV}$ X-ray peak.

A third case is provided by the limb flare from the same active region a few hours later, with a peak in the RHESSI 20$50 \mathrm{keV}$ emission at 09:29:48 UT The $\mathrm{H} \alpha$ line centre emission from the K5 kernel shows a dip over the period of the X-ray maximum, indicating absorption by chromospheric material, but the line wings show a maximum which is $\sim 17 \mathrm{~s}$ later than the 20-50 keV X-ray emission. Within the $\mathrm{H} \alpha$ line centre dip there is a small but significant peak which is $\sim 6 \mathrm{~s}$ later than the X-ray peak. 


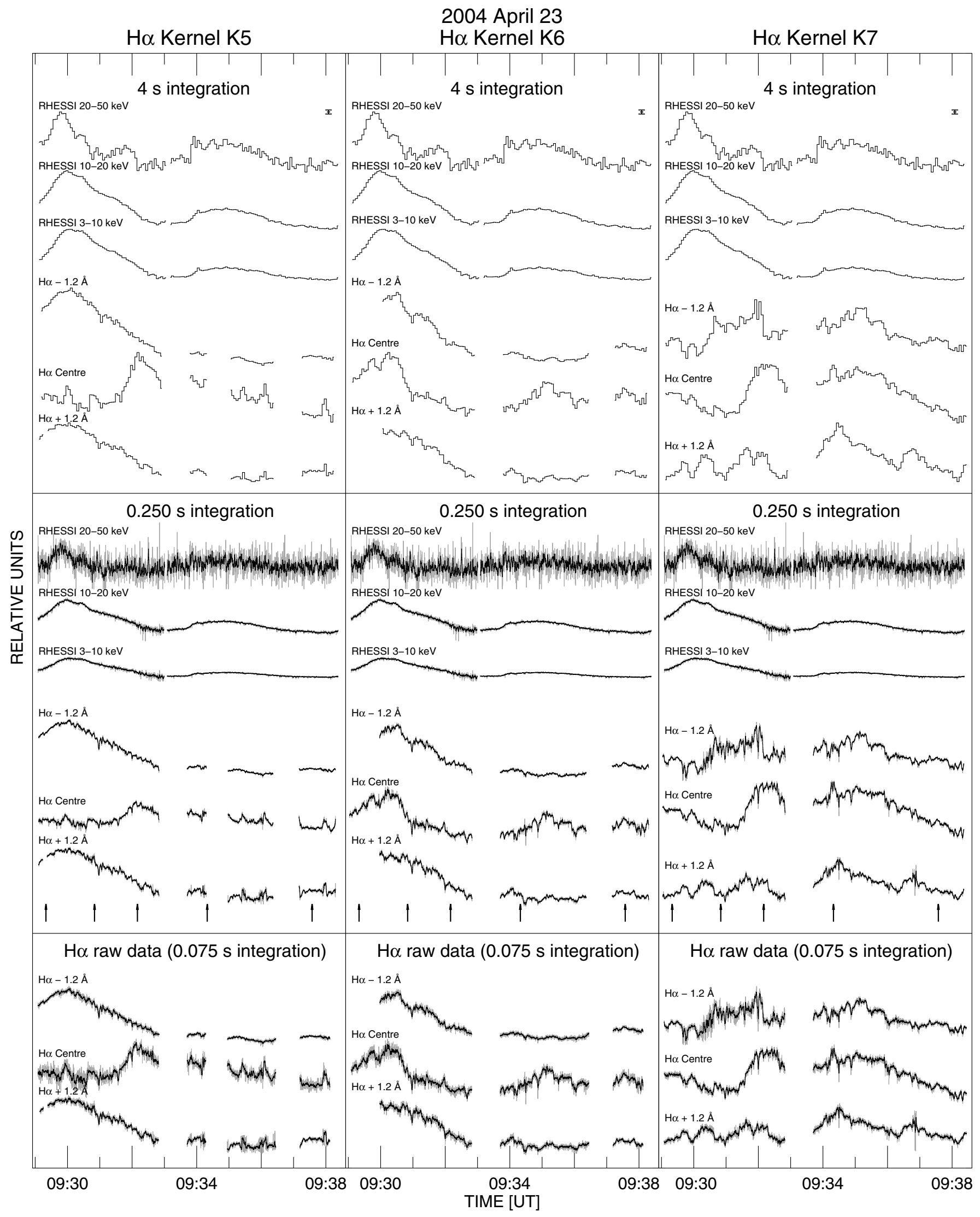

Fig. 9. The time-series of the RHESSI X-ray and LC-MSDP-SECIS $\mathrm{H} \alpha$ fluxes of the flaring kernels $\mathrm{K} 5$, $\mathrm{K} 6$ and emission sources $\mathrm{K} 7$ (shown in the three columns of the figure) recorded during the C4.4 flare in NOAA 10597 active region at 09:30 UT on 2004 April 23. The observations are arranged as in Fig. 7. The gap in the RHESSI data at about 09:33 UT is due to a change of the attenuation from A1 (thin attenuator) to A0 (no attenuator); this explains the decrease in scatter as the count rate is higher in the A0 state. The raw $\mathrm{H} \alpha$ data were taken with $0.075 \mathrm{~s}$ time resolution. Error bars are the same as in Fig. 7. The vertical arrows indicate the times of the flare images shown in Fig. 5. 


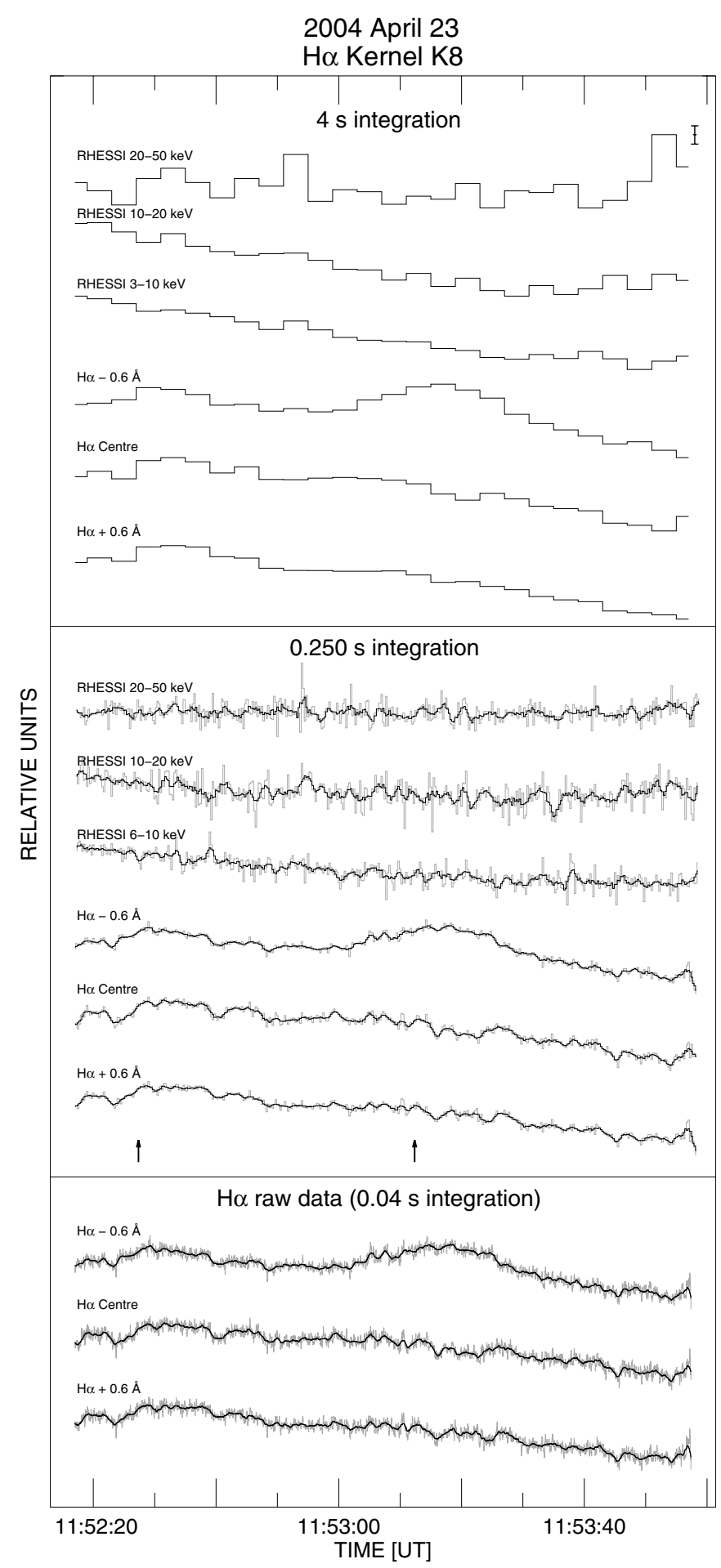

Fig. 10. The time-series of the RHESSI X-ray and LC-MSDP-SECIS $\mathrm{H} \alpha$ fluxes of the K8 flaring kernel, recorded about two minutes after maximum of the M1.5 flare in NOAA 10597 active region on 2004 April 23. The raw $\mathrm{H} \alpha$ data were taken with $0.04 \mathrm{~s}$ time resolution. The observations are arranged as in Fig. 7. Error bars are the same as in Fig. 7. The vertical arrows indicate the times of the flare images shown in Fig. 6.

The flares in which these correlations occurred are small and simple, and in our view allow a better study of the correlation of hard X-ray and $\mathrm{H} \alpha$ emission than in large, complex flares with possibly many impulsive bursts in different locations.
Broadly similar lag times of the $\mathrm{H} \alpha$ emission peaks compared with those in hard X-ray emission, particularly those observed for the $\mathrm{K} 1$ and $\mathrm{K} 4$ kernels $(\sim 3 \mathrm{~s}$ and $\sim 2 \mathrm{~s})$, have been observed before, e.g. Wang and co-workers (2000) found $\mathrm{H} \alpha$ flare emission to lag hard X-rays by $2-3 \mathrm{~s}$, Kurokawa with coworkers (1988) found the lag to be $\sim 1 \mathrm{~s}$, and Trottet with coworkers (2000) found the $\mathrm{H} \alpha$ and X-ray emission to be "nearly coincident". The time lag for the K5 kernel, either $\sim 17 \mathrm{~s}$ (line wings) or $\sim 6 \mathrm{~s}$ (line centre), are a little longer.

We may compare our results with the several theoretical studies that have been made of the chromospheric response to intense beams of nonthermal electrons accelerated at the flare impulsive stage and the consequent intensity of the $\mathrm{H} \alpha$ line emission. Using a set of dynamic model atmospheres and solving NLTE radiative transfer equations, Canfield \& Gayley (1987) found $\mathrm{H} \alpha$ line profiles with rapid response to a single pulse of energy resulting from an input electron beam. The emission in the $\mathrm{H} \alpha$ blue wing was found to be slightly delayed (up to $1 \mathrm{~s}$ ) because of ionization imbalance. Karlický (1990) has similarly studied the response of a model atmosphere which is heated by a single electron beam pulse using hydrodynamic and energy balance equations. The work of Heinzel (1991) builds on this. He found that the response of the $\mathrm{H} \alpha$ source function to very short $(<1 \mathrm{~s})$ pulses was first a dip then an enhancement lasting approximately $1 \mathrm{~s}$ for a large range of chromospheric hydrogen number densities, $n_{\mathrm{H}} \sim 10^{13}-10^{15} \mathrm{~cm}^{-3}$. For the densities of the order of $n_{\mathrm{H}} \sim 5 \times 10^{13}-10^{14} \mathrm{~cm}^{-3}$ a gradual increase of $\mathrm{H} \alpha$ emission can be observed during several pulses, while for higher densities $\left(n_{\mathrm{H}} \sim 10^{14}-10^{15} \mathrm{~cm}^{-3}\right)$ the $\mathrm{H} \alpha$ intensity impulsively increases. Later calculations (Karlický et al. 2004; Kasparová et al. 2005) are an improvement on this work, including, e.g., the effects of a return current associated with the electron beams, but their conclusions are very similar.

Our observations of small time lags, 2-3 s, in the $\mathrm{H} \alpha$ line emission with respect to hard (20-50 keV) X-ray emission for the $\mathrm{K} 1$ and $\mathrm{K} 4$ kernels are clearly compatible with the models of Heinzel (1991) and others. This assumes that the hard X-ray variations seen by RHESSI are a proxy for electron beam pulses.

We note that conduction fronts passing down flux loops following the initiation of energy release at the loop top would give rise to slightly longer delay times of the $\mathrm{H} \alpha$ emission. The conduction front velocity is approximately that of the ion sound speed, $\sim\left(1.5 k_{\mathrm{B}} T / m_{\mathrm{p}}\right)^{1 / 2}$ (where $T=$ the electron temperature of the plasma in the loop, $k_{\mathrm{B}}$ Boltzmann's constant, and $m_{\mathrm{p}}$ the proton mass). For $T=20 \mathrm{MK}$, the ion sound speed is $\sim 1000 \mathrm{~km} \mathrm{~s}^{-1}$. For a loop length of $15000 \mathrm{~km}$, as suggested by the images in Figs. 2-6, time lags of $\sim 15 \mathrm{~s}$ can be expected. Possibly such a time lag would apply to the observation of the K5 kernel in the 09:30 UT flare on 2004 April 23, but for the much smaller time lags of the $\mathrm{K} 1$ and $\mathrm{K} 4$ kernels the conduction front model is inappropriate.

In summary, we have used our equipment, a combination of the $L C, M S D P$ spectrograph, and the SECIS fast-frame CCD cameras, to observe the $\mathrm{H} \alpha$ line in nine wavelength bands across its profile during four small flares. Our observations, lasting several minutes in each case, have a time resolution as fine as $0.04 \mathrm{~s}$, better than what has been achieved in any previous study. This has allowed the detailed comparison of the $\mathrm{H} \alpha$ emission at various wavelength positions across the line profile to be compared with high-time-resolution observations made in X-rays with RHESSI. Comparison of $\mathrm{H} \alpha$ and hard (20-50 keV) emission for three small kernels K1, K4 and K5 indicates that the $\mathrm{H} \alpha$ emission lags the X-ray emission by $\sim 2-3 \mathrm{~s}(\mathrm{~K} 1, \mathrm{~K} 4)$, and either $\sim 17 \mathrm{~s}$ (line wings) or $\sim 6 \mathrm{~s}$ (line centre) for the K5 kernel. The 

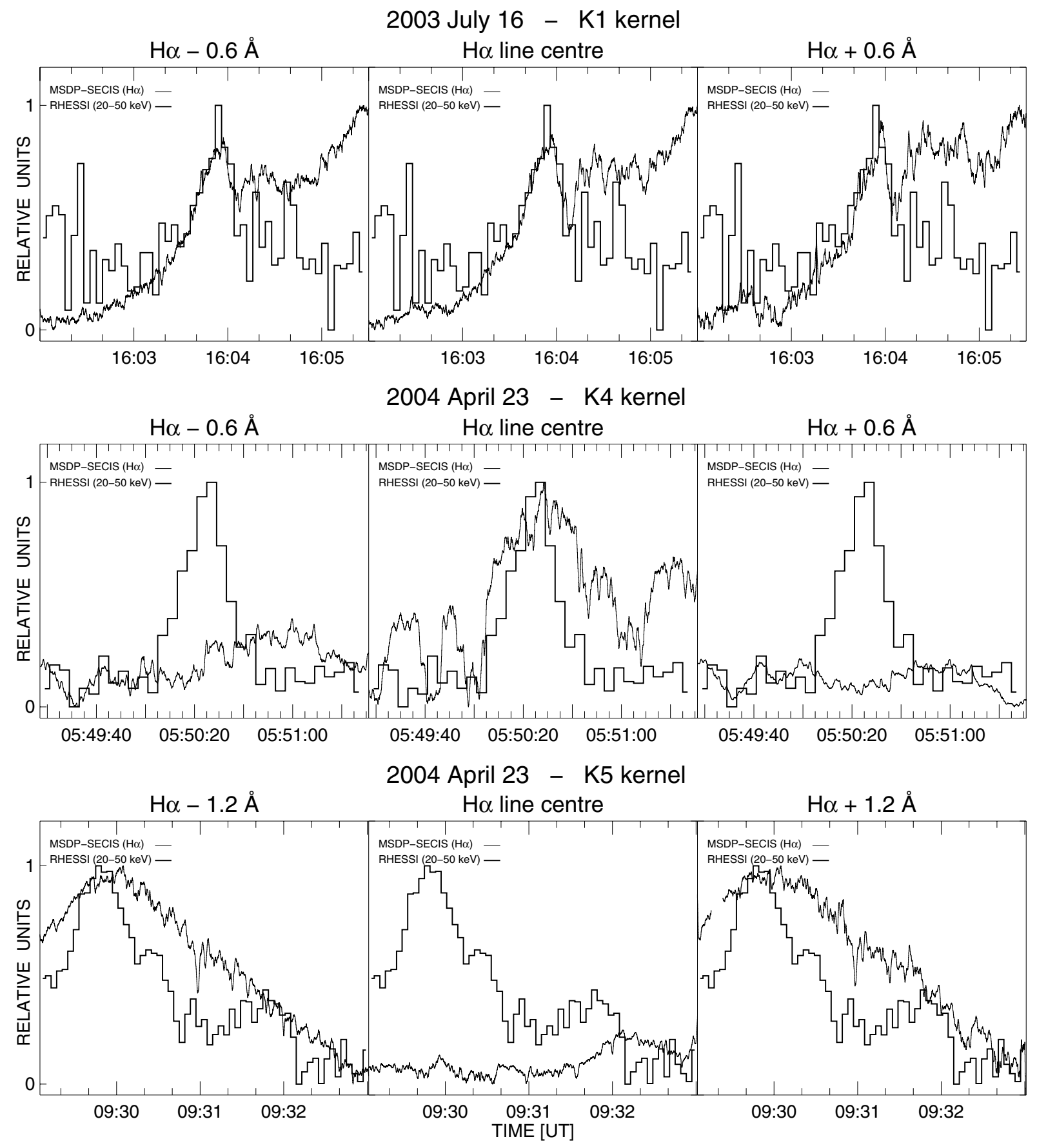

Fig. 11. Three examples of $\mathrm{H} \alpha$ light curves taken in the line centre and wings (LC-MSDP-SECIS data) and corresponding X-ray fluxes (20-50 keV $\mathrm{X}$-ray RHESSI data) showing time correlations. Upper row: X-ray and $\mathrm{H} \alpha$ light-curves (line centre and $\pm 0.6 \AA$ ) of the flaring kernel $\mathrm{K} 1 \mathrm{recorded}$ during the $\mathrm{C} 1.2$ flare in NOAA active region 10410 on 2003 July 16. Middle row: X-ray and $\mathrm{H} \alpha$ light-curves (line centre and $\pm 0.6 \AA$ ) of the flaring kernel K4 recorded during the B9.1 flare in NOAA active region 10597 on 2004 April 23. Bottom row: X-ray and H $\alpha$ light-curves (line centre and $\pm 1.2 \AA$ ) of the flaring kernel K5 recorded during the C4.4 flare in NOAA active region 10597 on 2004 April 23. The X-ray light curves are plotted logarithmically while the $\mathrm{H} \alpha$ data are shown using a linear scale. The variation ranges of the all light curves were scaled to unity except $\mathrm{H} \alpha \pm 0.6 \AA$ of the K4 kernel and $\mathrm{H} \alpha$ line centre for K5 kernel, where the observed signals were scaled to the quiet chromosphere. Supplementary information is given in captions of Figs. 7, 8 and 9.

values for the $\mathrm{K} 1$ and $\mathrm{K} 4$ kernels are in agreement with previous observations, and are compatible with electron beam models of Heinzel (1991) and others in which the electron beam is incident on chromospheric material. Possibly a conduction front model can explain the time lag of the K5 kernel.

In future work (Paper II), we will analyze data for several more flares observed in 2004-2005 and investigate the correlation of $\mathrm{H} \alpha$ emission (observed with the LC-MSDP-SECIS set-up) with RHESSI X-ray emission.

Acknowledgements. We are grateful to G. J. Hurford for kindly providing the RHESSI de-modulation software and many helpful remarks, and for advice on RHESSI count rate uncertainties by R. A. Schwartz. P.R. was supported by the Polish Ministry of Science and High Education grant N203 022 31/2991. K.J.H.P. thanks the National Research Council for the award of a Senior Research Associateship award at NASA Goddard Space Flight Center for some 
of the period of this research. K.R. is grateful to B. R. Dennis for financial support during a visit to the NASA Goddard Space Flight Center, USA. K.J.H.P. thanks the Leverhulme Trust for a travel grant from the UK to Poland. The continued loan of the SECIS cameras from the Rutherford Appleton Laboratory is acknowledged. We are very grateful to the referee for many helpful remarks and comments during the revision of the paper.

\section{References}

Canfield, R. C., \& Gayley, K. G. 1987, ApJ, 322, 999

Hanaoka, Y., Sakurai, T., Noguchi, M., \& Ichimoto, K. 2004, Adv. Space Res., 34,2753

Heinzel, P. 1991, Sol. Phys., 135, 65

Heinzel, P. 2003, Adv. Space Res., 32, 2393

Hudson, H. S., Strong, K. T., Dennis, B. R., et al. 1994, 1994, ApJ, 422, L25

Hurford, G. J., Schmahl, E. J., Schwartz, R. A., et al. 2002, Sol. Phys., 210, 61

Hurford, G. J. 2004, private communication

Karlický, M. 1990, Sol. Phys., 130, 347

Karlický, M., Kasparová, J., \& Heinzel, P. 2004, A\&A, 416, L13

Kasparová, J., Varady, M., Karlický, M., Moravec, Z., \& Heinzel, P. 2005, Presented at the European Solar Physics Division Meeting, Leuven, Belgium
Kitahara, T., \& Kurakawa, H. 1990, Sol. Phys., 125, 321

Kurokawa, H. 1983, Sol. Phys., 86, 195

Kurokawa, H., Kitahara, T., Nakai, Y., Funakoshi, Y., \& Ichimoto, K. 1986, Astrophys. Space Sci., 118, 149

Kurokawa, H., \& Takukura, T., \& Ohki, K. 1988, PASJ, 40, 357

Lin, R. P., Feffer, P. T., Schwartz, R. A. 2001, ApJ, 557, L125

Lin, R. P., Dennis, B. R., Hurford, G. J., et al. 2002, Sol. Phys., 210, 3

McAteer, R., Gallagher, P. T., Brown, D., et al. 2005, ApJ, 620, 1101

Mein, P. 1991, A\&A, 248, 669

Mrozek, T., \& Tomczak, M. 2004, A\&A, 415, 377

Phillips, K. J. H., Read, P. D., Gallagher, P. T., et al. 2000, Sol. Phys., 193, 259

Radziszewski, K., Rudawy, P., Phillips, K. J. H. 2005, 11th European Solar Physics Meeting, Leuven, Belgium, ESA CD: SP-600

Radziszewski, K., Rudawy, P., Phillips, K. J. H., \& Dennis, B. R. 2006, Adv. Space Res., 37, 1317

Rompolt, B., Mein, P., Mein, N., Rudawy, P., \& Berlicki, A. 1994, in JOSO Annual Report, ed. A. v. Alvensleben, 87

Rudawy, P., Phillips, K. J. H., Gallagher, P. T., et al. 2004, A\&A, 416, 1179

Trottet, G., Rolli, E., Magun, A., et al. 2000, A\&A, 356, 1067-1075

Wang, H., Qiu, J., Denker, C., et al. 2000, ApJ, 542, 1080 
K. Radziszewski et al.: High time resolution observations of solar H $\alpha$ flares. I., Online Material p 1

\section{Online Material}


K. Radziszewski et al.: High time resolution observations of solar H $\alpha$ flares. I., Online Material p 2

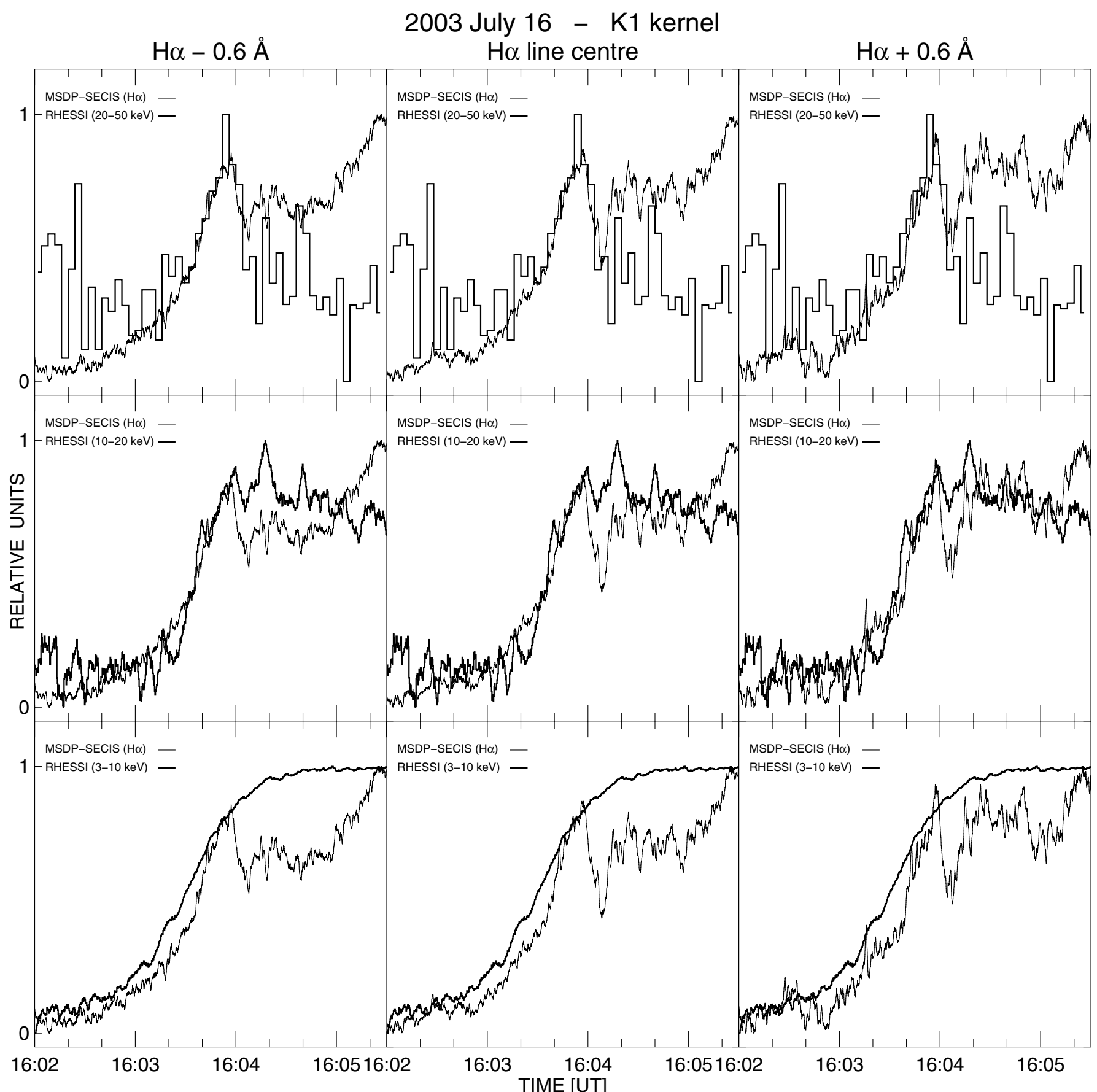

Fig.12. RHESSI X-ray and MSDP-SECIS $\mathrm{H} \alpha$ fluxes of the K1 flaring kernel recorded during the C1.2 flare on $2003 \mathrm{July} 16 . \mathrm{The} \mathrm{H} \alpha$ data, collected with $0.05 \mathrm{~s}$ time resolution and smoothed with a $1 \mathrm{~s}$ box-car filter, are shown for line centre and $\pm 0.6 \AA$ line wings in a linear scale. RHESSI data are plotted logarithmically in the energy ranges 3-10 keV (bottom row), 10-20 keV (middle row) and 20-50 keV (upper row). The integration times of the X-ray data are equal to $4 \mathrm{~s}$ in upper row and to $0.25 \mathrm{~s}$ in both lower rows (data are de-modulated and smoothed with $4 \mathrm{~s}$ box-car). The vertical axes have arbitrary units. See main text for details. 
K. Radziszewski et al.: High time resolution observations of solar H $\alpha$ flares. I., Online Material p 3

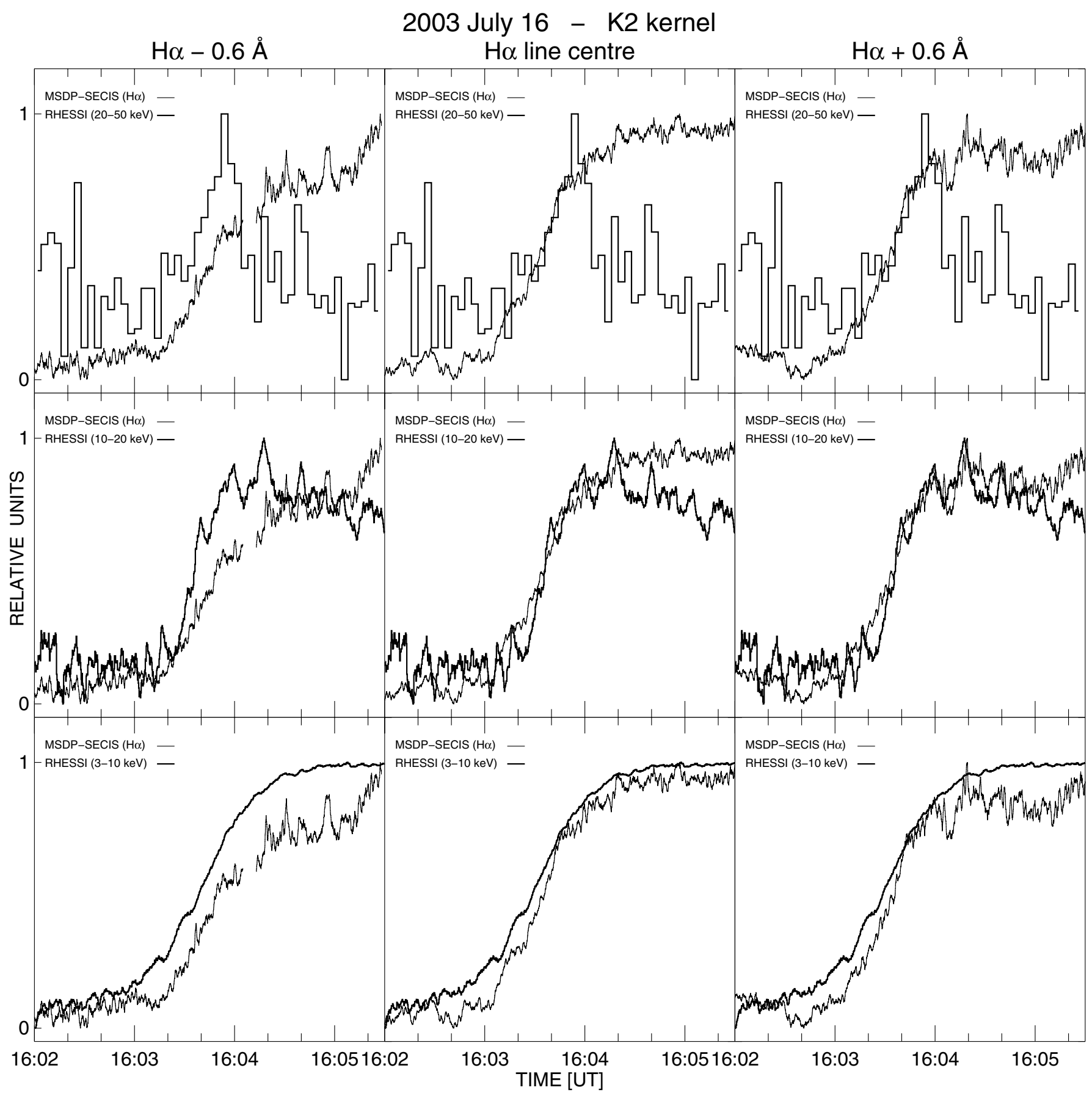

Fig.13. RHESSI X-ray and MSDP-SECIS $\mathrm{H} \alpha$ fluxes of the K2 flaring kernel recorded during the $\mathrm{C} 1.2$ flare on $2003 \mathrm{July} 16 . \mathrm{The} \mathrm{H} \alpha$ data, collected with $0.05 \mathrm{~s}$ time resolution and smoothed with a $1 \mathrm{~s}$ box-car filter, are shown for line centre and $\pm 0.6 \AA$ line wings in a linear scale. RHESSI data are plotted logarithmically in the energy ranges 3-10 keV (bottom row), 10-20 keV (middle row) and 20-50 keV (upper row). The integration times of the X-ray data are equal to $4 \mathrm{~s}$ in upper row and to $0.25 \mathrm{~s}$ in both lower rows (data are de-modulated and smoothed with $4 \mathrm{~s}$ box-car). The vertical axes have arbitrary units. See main text for details. 
K. Radziszewski et al.: High time resolution observations of solar H $\alpha$ flares. I., Online Material p 4

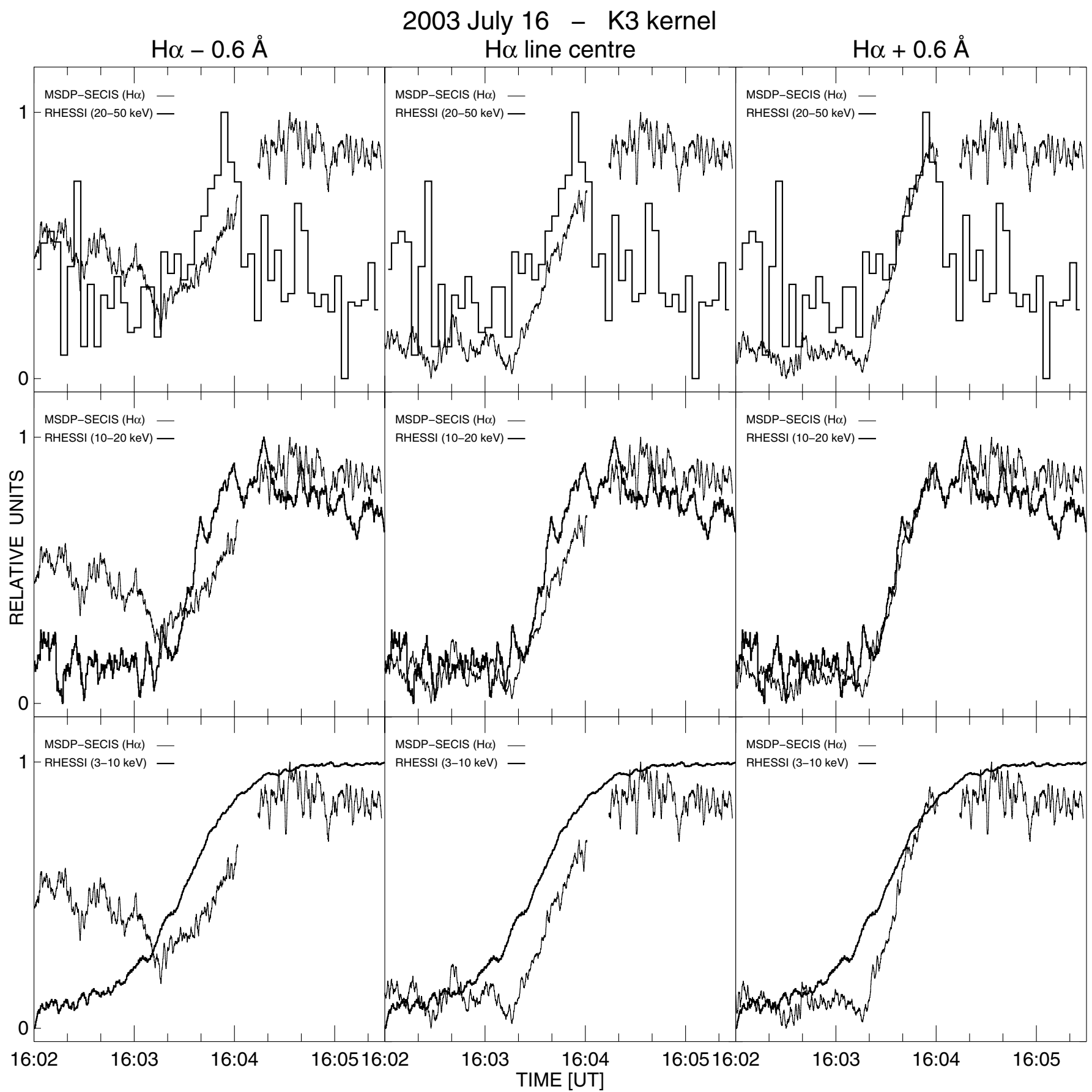

Fig.14. RHESSI X-ray and MSDP-SECIS $\mathrm{H} \alpha$ fluxes of the K3 flaring kernel recorded during the $\mathrm{C} 1.2$ flare on $2003 \mathrm{July} 16 . \mathrm{The} \mathrm{H} \alpha$ data, collected with $0.05 \mathrm{~s}$ time resolution and smoothed with a $1 \mathrm{~s}$ box-car filter, are shown for line centre and $\pm 0.6 \AA$ line wings in a linear scale. RHESSI data are plotted logarithmically in the energy ranges 3-10 keV (bottom row), 10-20 keV (middle row) and 20-50 keV (upper row). The integration times of the X-ray data are equal to $4 \mathrm{~s}$ in upper row and to $0.25 \mathrm{~s}$ in both lower rows (data are de-modulated and smoothed with $4 \mathrm{~s}$ box-car). The vertical axes have arbitrary units. See main text for details. 
K. Radziszewski et al.: High time resolution observations of solar H $\alpha$ flares. I., Online Material p 5

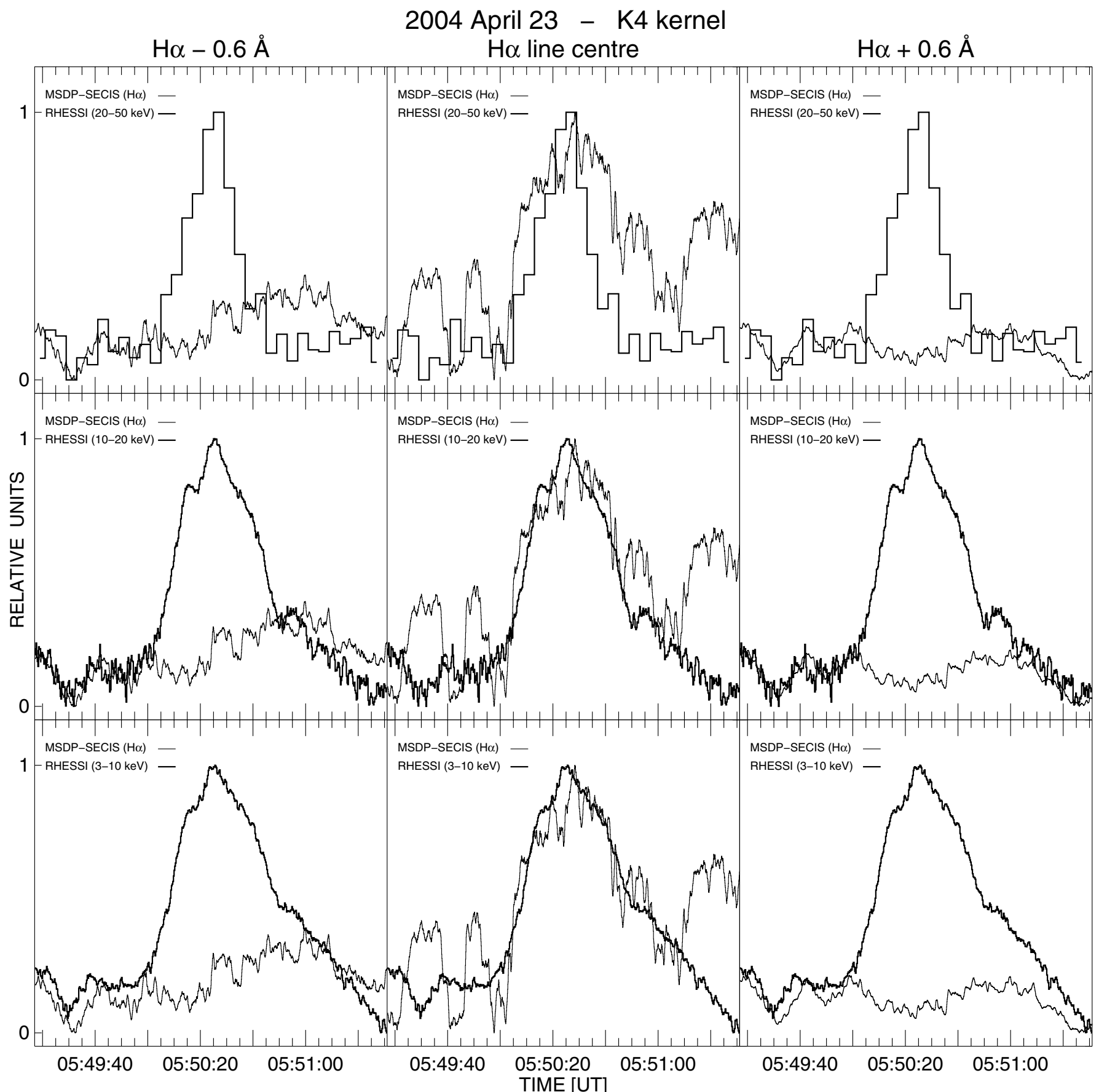

Fig.15. RHESSI X-ray and MSDP-SECIS $\mathrm{H} \alpha$ fluxes of the $\mathrm{K} 4$ flaring kernel recorded during the B9.1 flare on $2004 \mathrm{April} 23$. The $\mathrm{H} \alpha$ data, collected with $0.075 \mathrm{~s}$ time resolution and smoothed with a $1 \mathrm{~s}$ box-car filter, are shown for line centre and $\pm 0.6 \AA$ line wings in a linear scale. RHESSI data are plotted logarithmically in the energy ranges 3-10 keV (bottom row), 10-20 keV (middle row) and 20-50 keV (upper row). The integration times of the X-ray data are equal to $4 \mathrm{~s}$ in upper row and to $0.25 \mathrm{~s}$ in both lower rows (data are de-modulated and smoothed with $0.5 \mathrm{~s}$ box-car). The vertical axes have arbitrary units. See main text for details. 
K. Radziszewski et al.: High time resolution observations of solar H $\alpha$ flares. I., Online Material p 6

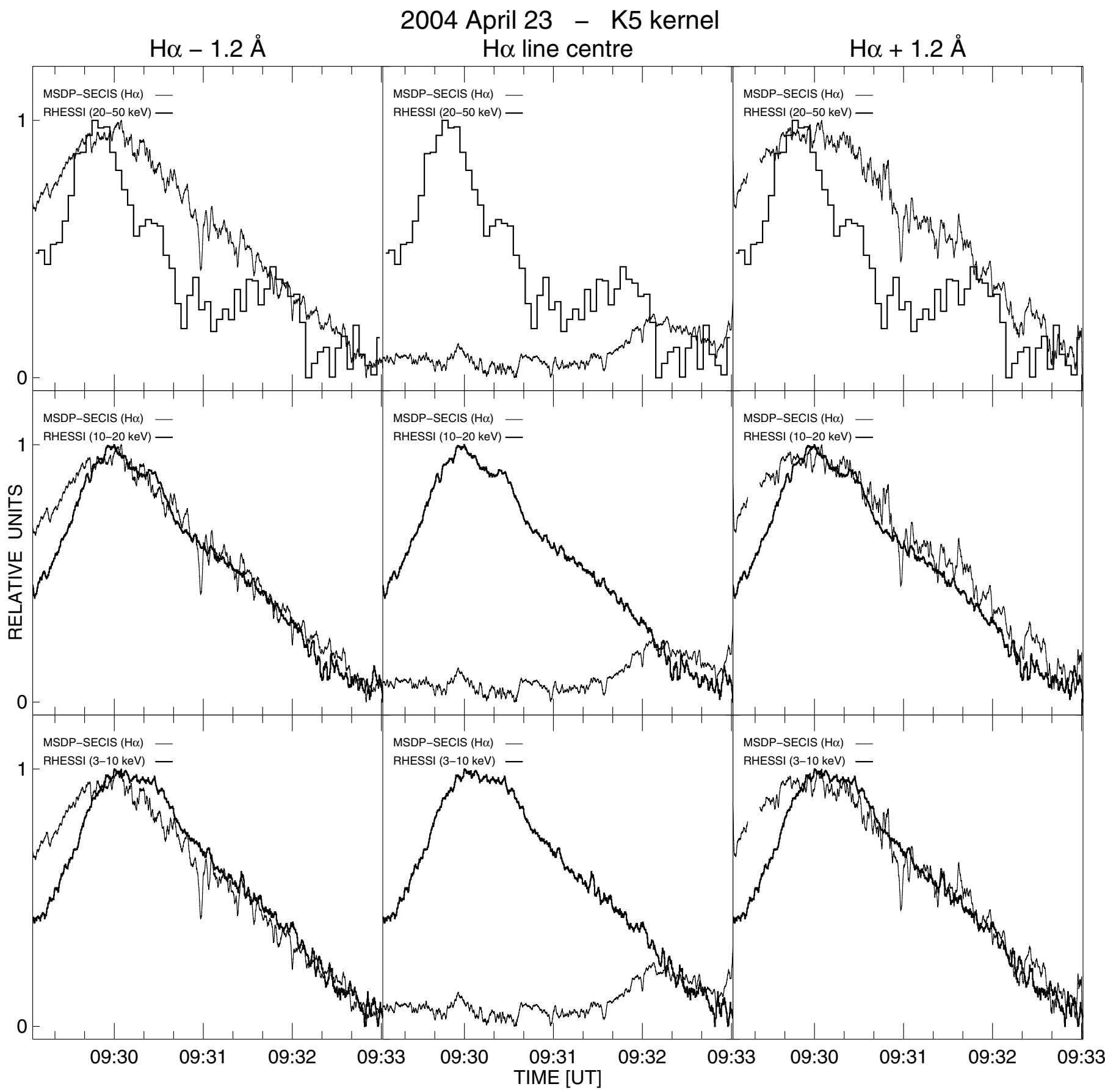

Fig.16. RHESSI X-ray and MSDP-SECIS H $\alpha$ fluxes of the K5 flaring kernel recorded during the C4.4 flare on 2004 April 23. The H $\alpha$ data, collected with $0.075 \mathrm{~s}$ time resolution and smoothed with a $1 \mathrm{~s}$ box-car filter, are shown for line centre and $\pm 1.2 \AA$ line wings in a linear scale. RHESSI data are plotted logarithmically in the energy ranges 3-10 keV (bottom row), 10-20 keV (middle row) and 20-50 keV (upper row). The integration times of the X-ray data are equal to $4 \mathrm{~s}$ in upper row and to $0.25 \mathrm{~s}$ in both lower rows (data are de-modulated and smoothed with $2 \mathrm{~s}$ box-car). The vertical axes have arbitrary units. See main text for details. 
K. Radziszewski et al.: High time resolution observations of solar H $\alpha$ flares. I., Online Material $p 7$

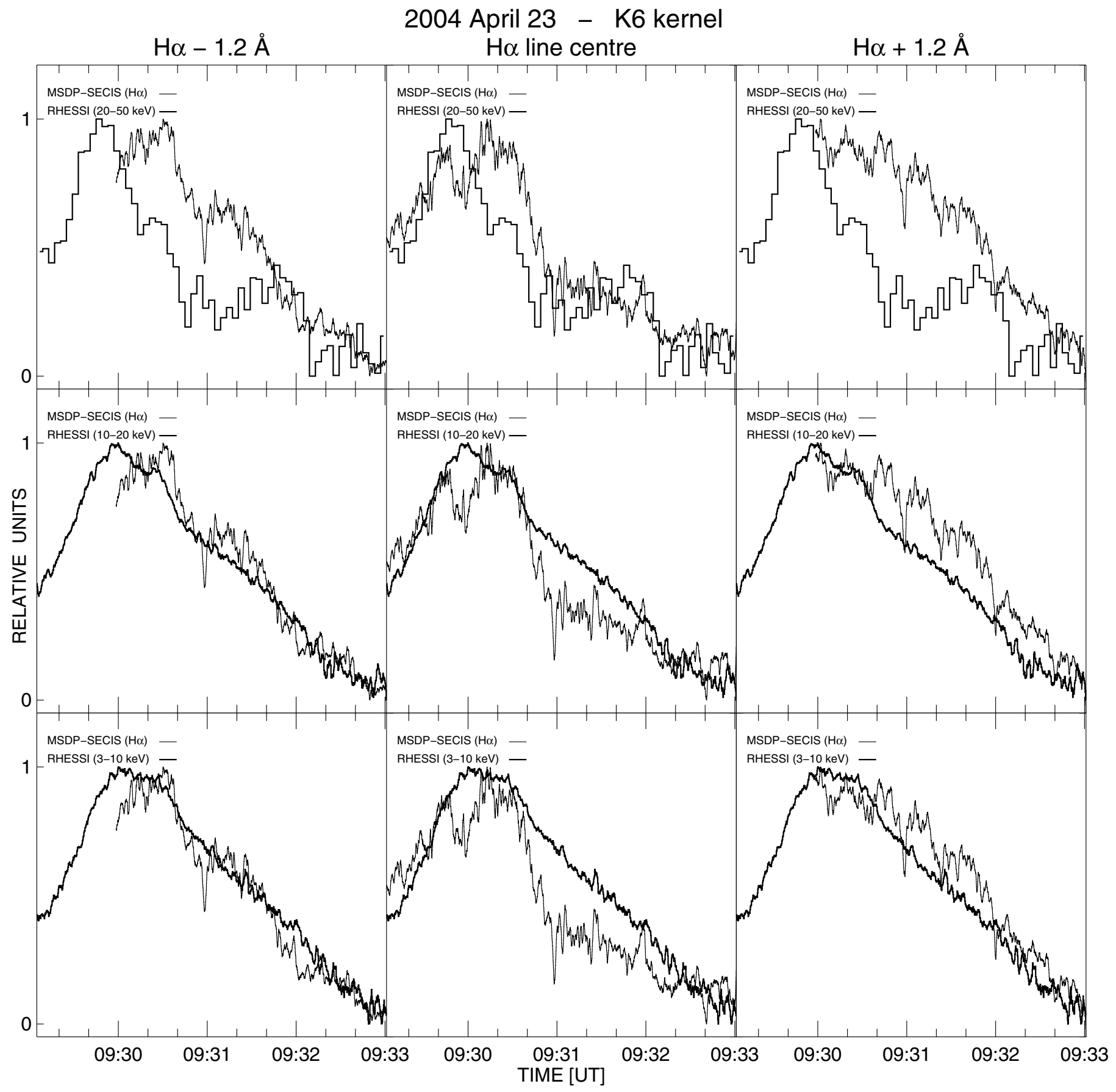

Fig.17. RHESSI X-ray and MSDP-SECIS H $\alpha$ fluxes of the K6 flaring kernel recorded during the C4.4 flare on 2004 April 23. The H $\alpha$ data, collected with $0.075 \mathrm{~s}$ time resolution and smoothed with a $1 \mathrm{~s}$ box-car filter, are shown for line centre and $\pm 1.2 \AA$ line wings in a linear scale. RHESSI data are plotted logarithmically in the energy ranges 3-10 keV (bottom row), 10-20 keV (middle row) and 20-50 keV (upper row). The integration times of the X-ray data are equal to $4 \mathrm{~s}$ in upper row and to $0.25 \mathrm{~s}$ in both lower rows (data are de-modulated and smoothed with $2 \mathrm{~s}$ box-car). The vertical axes have arbitrary units. See main text for details. 